Franz Leander Fillafer, Thomas Wallnig (Hg.)

\title{
Josephinismus zwischen den Regimen
}

Eduard Winter, Fritz Valjavec und die zentraleuropäischen

Historiographien im 20. Jahrhundert

\section{ELEKTRONISCHER \\ SONDERDRUCK}

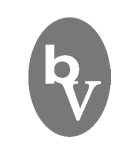

2016

BÖHLAU VERLAG WIEN · KÖLN · WEIMAR 


\section{Auftrag und Erfüllung. Erna Lesky und medizinhistorische Narrative im 20. Jahrhundert}

\section{EINLEITUNG}

Die folgenden Ausführungen verbinden die Geschichte der Medizin in Wien mit der Geschichte der Medizingeschichtsschreibung und spitzen die Analyse auf die Funktion des „Josephinismus" in beiden Bereichen zu. Eine umfassende Einbettung der präsentierten Ergebnisse zu Lehrinhalten der medizinischen Ausbildungsstätten sowie zu Überformungen und Methodenproblemen der Medizinhistoriographie kann hier nicht geboten werden, daher bleibt vor allem die wissenschaftsgeschichtliche Kontextualisierung der Wiener Medizingeschichtsschreibung mit ihren persönlichen Beziehungsnetzwerken und ihrer Einbindung in international relevante Entwicklungen hier nur vorläufig und skizzenhaft.

In der vorliegenden Analyse geht es auch nicht um Erna Lesky und ihre Biographie, nicht um die Bewertung ihrer Leistungen und ihrer Karriere als erste Ordinaria an der medizinischen Fakultät der Universität Wien und auch nicht um eine Diskussion ihres Verhältnisses zum Nationalsozialismus als Ärztin in einem Tiroler NSV-Mutter-und-Kind-Heim. Dies bleibt jenen vorbehalten, die sich biographisch mit ihrer Lebenszeit beschäftigen. ${ }^{1}$

1 Erna Lesky (geb. Klingenstein, 1911-1986) studierte zunächst Medizin und war in Innsbruck als Kinderärztin tätig. Sie war mit dem Altphilologen Albin Lesky verheiratet, der ab 1936 Professor für Klassische Philologie an der Universität Innsbruck, ab 1949 an der Universität Wien war und 1963/64 auch das Amt des Rektors bekleidete. Albin Lesky war Mitglied der NSDAP, 1963-1969 Vizepräsident und 1969/70 Präsident der Österreichischen Akademie der Wissenschaften. Erna Lesky widmete sich nach ihrer Heirat verstärkt der Medizingeschichte, erwarb das Doktorat der Geschichte und habilitierte sich 1957 im Fach „Geschichte der Medizin“, am selben Tag wie Marlene Jantsch (1917-1994), die sich in der Nachkriegszeit dem Wiederaufbau des Instituts für Geschichte der Medizin gewidmet hatte. 1960 übernahm Erna Lesky die Leitung des Instituts für Geschichte der Medizin, 1966 wurde sie zur ordentlichen Professorin ernannt, der ersten an der Wiener medizinischen Fakultät. Vgl. u. a. Michael Hubenstorf, Von Erfolg und Tragik einer Medizinhistorikerin, Erna Lesky (1911-1986). In: Christoph Meinel / Monika RenNEBERG (Hg.), Geschlechterverhältnisse in Medizin, Naturwissenschaft und Technik. Im Auftrag des Vorstandes der Deutschen Gesellschaft für Geschichte der Medizin, Naturwissenschaft und Technik. Bassum 1996, 98-109; Helmut Gröger, Lesky, Erna, geb. Klingenstein. In: Brigitta KeIntZel / Ilse KorotiN (Hg.), Wissenschaftlerinnen in und aus Österreich. Leben - Werk - Wirken. Wien 2002, 465-468. Zur Karrierestrategie von Erna Lesky: Felicitas Seebacher, Erna Lesky, General and Diplomat. Networking as a Power Tool for the History of Medicine. In: Antonio Roca-Rosell (Hg.), The Circulation of Science and Technology. Proceedings of the 4th International Conference of the European Society for the History of Science, Barcelona, 18-20 November 2010. Barcelona 2012, 208-216. 
Der Konzeption des Bandes entsprechend geht es auch in diesem Beitrag um die Wahrnehmung und Interpretation dessen, was als "Josephinismus“ in die österreichische Geschichtsschreibung Eingang gefunden hat, im Speziellen im Hinblick auf die Arbeiten von Fritz Valjavec und Eduard Winter, und hier im Besonderen auf die Frage der Medizingeschichtsschreibung, wie sie von Erna Lesky vertreten wurde. Daraus ergibt sich auch für eine Re-Lektüre ihrer Werke die Frage, „was“ und „wie“ über Josephinismus geschrieben wurde, konkret: in welcher Weise Medizin- und Gesundheitswesen - bei ihr und bei anderen - als Teil des Josephinismus aufgefasst wurden und somit das Verständnis dieser Epoche in der österreichischen Geschichtsschreibung mit bestimmen konnten.

Gesundheits-, Sozial- und Bildungswesen waren wesentliche Bereiche der gesellschaftspolitischen Maßnahmen der Regierung der habsburgischen Länder in der zweiten Hälfte des 18. Jahrhunderts. Diese Aspekte wurden bei Eduard Winter und Fritz Valjavec nur am Rande berücksichtigt, wie in Abschnitt VIII zu zeigen sein wird. Interessant ist freilich, dass Eduard Winter in seiner theologischen Habilitationsschrift, die er 1922 an der Universität Prag eingereicht hatte, eine „pastorale Lösung“ der zu diesem Zeitpunkt aktuellen Probleme im Gesundheits- und Sozialwesen der jungen Tschechoslowakei vorschlug. ${ }^{2}$ Basierend auf einer ausführlich dokumentierten und präzisen Analyse der Situation, wurde ein Konzept entworfen, das in seiner pastoral-christlichen Ausrichtung eine starke Ähnlichkeit mit jenem aufweist, das in den Habsburgischen Ländern während der Regierung von Joseph II. etabliert wurde und noch lange wirksam war.

In ihrer 1959 erschienenen Monographie Österreichisches Gesundheitswesen im Zeitalter des aufgeklärten Absolutismus ${ }^{3}$ thematisiert Erna Lesky einige Aspekte des Gesundheitswesens in der zweiten Hälfte des 18. Jahrhunderts und verwendet dabei jene Topoi, die bis heute wahrnehmungsleitend sind und kaum hinterfragt wurden. Zwei von ihnen, der Legendenbildung um Gérard van Swieten und dem Unterricht am Krankenbett, sind die Abschnitte V und VI gewidmet.

Mehrmals bezieht Lesky sich in dieser Publikation, aber auch in anderen Texten auf Eduard Winter und Fritz Valjavec, wenn es um allgemein gehaltene Bemerkungen zum Josephinismus geht. Die von ihr vertretenen Zugänge sind offenbar stark von deren Auffassungen geprägt, etwa im Hinblick auf die Rolle der Staatlichkeit, aber auch in Bezug auf das Thema „Nationalismus“, was im Folgenden erläutert wird. Insofern ruft besonders ihre Arbeit über das österreichische Gesundheitswesen den Eindruck hervor, eine Art Ergänzung zu den Arbeiten von Valjavec und Winter zu sein; jedenfalls fügt sich diese in das von den beiden Historikern entworfene Bild des Josephinismus ein.

Werden die von Lesky verwendeten Topoi jedoch hinterfragt und wird vor allem deren historiographisches Fundament analysiert, erlaubt dies auch eine Einschätzung der Summe an politischen, kulturellen, intellektuellen und sozialen Entwicklungen, die unter dem Be-

2 Eduard Winter, Die Gesundheitsfürsorge auf dem Lande als Ausgangs- und Mittelpunkt der ländlichen Wohlfahrtspllege in ihrer pastoralen und sozialen Bedeutung (Prag 1922), hg. von Hildegard РAutsch in freundschaftlicher Würdigung der Persönlichkeit Eduard Winter. Salzburg 1984.

3 Erna Lesky, Österreichisches Gesundheitswesen im Zeitalter des aufgeklärten Absolutismus. Wien 1959. 
griff ,Josephinismus“ zusammengefasst wurden. Dieser Zugang ermöglicht es zudem, Aspekte aufzuzeigen, die bei dieser Konstruktion des „Josephinismus" unberücksichtigt geblieben sind oder eine einseitige Interpretation erfahren haben.

Der Auswahlprozess, durch den der Josephinismus auf sein Bedeutungsspektrum festgelegt wurde, beginnt in der Medizingeschichtsschreibung jedoch nicht mit Erna Lesky. Im Folgenden wird skizziert, dass die zentralen Topoi in Leskys master narrative des Gesundheitswesens der Habsburgischen Länder in der zweiten Hälfte des 18. Jahrhunderts als Kulminationspunkte einer längeren institutionellen Entwicklung zu sehen sind. Durch die in Wien immerhin seit dem späten 18. Jahrhundert existierende institutionalisierte Medizingeschichtsschreibung erfolgte eine Auswahl dessen, was an gesundheits- und sozialpolitischen Maßnahmen thematisiert wurde. So traten einige Aspekte besonders deutlich hervor, andere, so scheint es, sind einer Art damnatio memoriae, zumindest aber einer zweckdienlichen Interpretation anheimgefallen. Diese historiographie- und institutionengeschichtliche Diskussion erfolgt in den Abschnitten II bis IV.

Ähnliche Vorgänge der Auswahl, Kanonisierung und Instrumentalisierung könnten auch bei anderen Themen und in der ,allgemeinen Geschichtsschreibung " über diese Epoche erfolgt sein. Mehrere Beiträge des vorliegenden Bandes weisen solche Vorgänge in den Werken von Valjavec und Winter nach. Die in ihren Texten angebotenen Definitionen von "Josephinismus“ könnten daher als Ergebnis früherer politischer Interpretationen, etwa aus der Zeit des Vormärz und der Restauration, verstanden werden, ${ }^{4}$ aber auch als Interpretationen, die - unhinterfragt oder auch mit Absicht - weitergetragen wurden. Medizin und Gesundheitswesen des "Josephinismus" sind jedenfalls aussagekräftige Beispiele für diesen Prozess. Sie erlauben eine Annäherung an die wohl von politischen Zielen getragene Rezeption wesentlicher Aspekte der politischen und gesellschaftlichen Veränderungen des ausgehenden 18. Jahrhunderts.

Wie sich diese Reformen im Lichte der jüngeren Forschung darstellen, soll der historiographischen Analyse vorangestellt werden, um die Kontraste besser herausarbeiten zu können.

\section{MEDIZIN UND GESUNDHEITSWESEN DES SPÄTEN I 8. JAHRHUNDERTS}

Die Medizin des späten 18. Jahrhunderts stand vor Herausforderungen, die in den zeitgenössischen politischen und wirtschaftlichen Rahmenbedingungen begründet lagen. Ein wesentliches Ziel war die Erhöhung der Bevölkerungszahlen, wobei es nicht um die Zahl alleine ging, sondern um die Vergrößerung einer gesunden, produktiven und konsumierenden Bevölkerung.

In ihrer grundlegenden Konzeption war die frühneuzeitliche Medizin auf die Behandlung des Individuums ausgerichtet. Zentrale Ansatzpunkte war in dieser Denkweise eine sehr genaue Wahrnehmung des aktuellen Zustandes des Menschen und eine darauf individuell abgestimmte Behandlung bzw. die Unterstützung einer die individuelle Gesundheit fördern-

4 Vgl. den Beitrag von Franz L. Fillafer in diesem Band. 
den Lebensführung. Wesentlich ist hierbei, dass medizinische Behandlungen nicht erst dann eingesetzt wurden, wenn ein Mensch bereits erkrankt war, sondern dass vorbeugende Maßnahmen Priorität hatten, um die Gesundheit möglichst lange zu erhalten. Insofern waren präventive Behandlungen und eine gesunde Lebensführung wesentliche Komponenten dieses medizinischen Konzeptes.

Im Krankheitsfall wurde der individuelle Zustand der Betroffenen zunächst genau beobachtet, analysiert und eine auf diesen Zustand abgestimmte Behandlung begonnen. Danach wurde überprüft, wie die Kranken auf diese ersten Maßnahmen reagierten. Dementsprechend wurde die Therapie bis zur Gesundung laufend an die individuellen Reaktionen angepasst. Medizinische Therapien inkludierten nicht nur die Anwendung von innerlich wirkenden Arzneimitteln, die so genannte cura interna, sondern auch Behandlungen, die primär über die Körperoberfläche wirken, die cura externa. Erstere wurde hauptsächlich von akademischen Heilkundigen angewandt, letztere umfasste meist chirurgische Maßnahmen und war der Arbeitsbereich von Badern und Wundärzten. Sowohl in der cura interna als auch in der cura externa wurde also mit den individuellen Zuständen von Patientinnen und Patienten gearbeitet und darauf abgestimmt vorgegangen. Dieses medizinische Denken beruhte auf einem elaborierten (und reichlich komplexen) Verständnis des menschlichen Körpers und körperlicher Vorgänge.

Die wirtschafts- und sozialpolitischen Ansprüche der werdenden modernen Staaten, die unter anderem eine hohe Bevölkerungszahl als Ziel verfolgten, stellten an das medizinische Denken Anforderungen, die über eine Auseinandersetzung mit dem Individuum hinaus gingen. Nunmehr sollten zahlreiche Individuen, nämlich die Bevölkerung als Ganzes, berücksichtigt werden. Dabei standen nicht nur der Umgang mit Erkrankungen im Vordergrund, die epidemisch oder endemisch auftraten und viele Menschen erfassten, sondern auch längerfristige und umfassende Maßnahmen, die die Gesundheit der Bevölkerung fördern sollten. Dem oben genannten Konzept entsprechend ging es im Wesentlichen um Prävention sowie um Möglichkeiten, frühzeitig in ein Krankheitsgeschehen einzugreifen. Eines der Ziele war daher der Aus- und Aufbau einer flächendeckenden, medizinischen Betreuung durch professionelle Heilkundige und die Sicherung des Zuganges zu medizinischer und sozialer Versorgung, auch in Regionen, in denen die notwendige Infrastruktur nicht oder nur begrenzt vorhanden war. Besonders relevant waren weitreichende präventive Maßnahmen wie die Impfung der gesamten Bevölkerung gegen die Pocken und die Schaffung von gesundheitsfördernden Lebensbedingungen. Dazu gehörte eine nach hygienischen Gesichtspunkten gestaltete Stadtplanung ebenso wie etwa die ausreichende Versorgung mit Lebensmitteln, die Kontrolle der Hygiene auf Märkten, die Vermittlung von medizinischem Wissen an die Bevölkerung und die exakte Dokumentation der Bevölkerungsentwicklung.

Grundsätzlich war bekannt, was in konkreten Fällen zu tun war, etwa im Bereich der Stadtplanung oder im Umgang mit Epidemien, ausschlaggebend war jedoch nunmehr, dass sämtlich Maßnahmen flächendeckend umgesetzt und deren Effizienz überprüfbar gemacht werden sollten. ${ }^{5}$

5 In Bezug zur Stadtplanung vgl. Markus SwitTalek, Das Josephinum. Aufklärung, Klassizismus, Zent- 
Das medizinische Wissen und Handeln war also vor die Herausforderung gestellt, zusätzlich zur Behandlung von Individuen auch Kenntnisse über die Förderung der Gesundheit der Allgemeinheit zu entwickeln, aber auch Handlungsweisen und Instrumente zur „Informationsgewinnung “ anzuwenden, um die ganze Bevölkerung - und eben nicht nur Einzelne - medizinisch beobachten und betreuen zu können. Hierbei ging es auch um die Frage der Effektivität von Maßnahmen, die sich in den Morbiditäts- und Mortalitätsraten abbilden ließen. Statistische Methoden wurden als Instrumente eingesetzt, um das Wachstum der Bevölkerung ebenso wie Krankheitshäufigkeiten und Sterblichkeit zu dokumentieren und beobachten zu können. Diese Instrumente zu Beobachtung der Bevölkerungsentwicklung waren keine neuen Erfindungen, vielmehr sind diese als political arithmetic besonders von John Graunt (1620-1674) und Willam Petty (1623-1687) im 17. Jahrhundert propagierte Instrumente, die dazu dienen sollten, die englische Wirtschaft zu stärken. Besondere Bedeutung wird diesen Beobachtungen der Bevölkerungsentwicklung im Werk Medizinische Polizey von Johann Peter Frank (1745-1821) zugeschrieben. ${ }^{6}$ Hierbei beruft Frank sich wiederum auf Konzepte und Messmethoden, die auf Johann Peter Süßmilch (1707-1767) und Kaspar Neumann (1648-1715) zurückgehen.

Um diesen Anforderungen entsprechen zu können, musste auch das notwendige Wissen produziert und vermittelbar gemacht werden, was in der Folge zur Entwicklung von neuen medizinischen Fächern mit einem definierten Wissenskanon führte, wie etwa der „Medizinischen Polizey“ und der "Staatarzneykunde“, die auch die forensische Medizin umfasste. Dass die Geburtshilfe in diesem Zusammenhang eine besondere Rolle spielte, versteht sich von selbst. Auch Gebiete der Heilkunde, die für den „Alltag“ der Bevölkerung von Relevanz waren, etwa die Dermatologie, die Augen- und die Zahnheilkunde, wurden als eigene Fächer definiert, vielfach erfolgte hierbei erstmals eine Verschriftlichung von bestehendem und bislang hauptsächlich mündlich und praktisch weiter gegebenem medizinischem Wissen. ${ }^{7}$ Dies bedeutet jedoch nicht, dass Wissen und Kenntnisse in diesen Bereichen nicht schon zuvor vorhanden gewesen wären, nunmehr erfolgte jedoch die Definition als Fachgebiet mit einem festgelegten Wissenskanon, mit speziellen Praktiken und Behandlungen sowie dem Zusammenfassen dieses Wissens in Lehrbüchern und nicht nur in medizinischer Expertenliteratur.

Offenbar gestaltete es sich jedoch schwierig, diese Zugänge an medizinischen Fakultäten durchzusetzen, wie die bislang untersuchten Beispiele von Wien und Köln/Bonn zeigen. ${ }^{8}$

rum der Medizin. Diss. TU Wien 2014. - Zum breiteren Kontext staatlicher Maßnahmen im Bereich des Gesundheitswesens und der Bevölkerungsentwicklung: Michael Hochedlinger / Anton TANTner (Hg.), ,... der größte Teil der Untertanen lebt elend und mühselig“": die Berichte des Hofkriegsrates zur sozialen und wirtschaftlichen Lage der Habsburgermonarchie 1770-1771, Innsbruck 2005.

6 Johann Peter Frank, System einer vollständigen medicinischen Polizey. 8 Bde., Mannheim u. a. 17791825.

7 Vgl. dazu: Theresa HüTter, Die „Materia Chirurgica“ von Joseph Jakob Plenk. Analyse eines chirurgischen Lehrbuches des 18. Jahrhunderts. Diplomarbeit MedUni Wien 2014.

8 Sonia Horn, „... reineres Licht über die Wissenschaften im Erzstifte zu Köln ...“. Medizin, Gesundheitswesen und Aufklärung an der Maxischen Akademie in Bonn und der medizinisch- chirurgischen 
Anzunehmen ist Ähnliches auch zumindest für Olmütz, in Kopenhagen und Edinburgh existierten neben den medizinischen Fakultäten ebenfalls parallele Institutionen zur medizinischen Ausbildung.

In Bonn und Wien wurden neben den bereits existierenden medizinischen Fakultäten neue medizinische Ausbildungszentren etabliert, die ebenfalls akademische Grade in der Medizin vergeben konnten, an denen jedoch einem anderen Curriculum gefolgt wurde als an den medizinischen Fakultäten. Die Universität Köln war 1381 mit einer medizinischen Fakultät gegründet worden. 1773 war im nahegelegenen Bonn, dem Sitz des Kölner Erzbischofs Maximilian Friedrich (1708-1784), nach der Aufhebung des Jesuitenkollegs eine Akademie eingerichtet worden, die „Maxische Akademie“, an der auch medizinische Fächer gelehrt wurden. Diese neue Akademie zeigte vor allem in philosophischen und sprachlichen Fächern eine personelle Kontinuität, zumal hier weiterhin Jesuiten tätig waren. In den medizinischen Bereichen wurde jedoch eindeutig das Ziel verfolgt, einen Wohlfahrtsstaat aufzubauen und somit Konzepte der Aufklärung umzusetzen, wie dies die relevanten Akteure selbst bezeichneten. 1784 wurde diese Institution durch ein Privileg von Joseph II. zu einer Universität, zu der auch eine medizinische Fakultät gehörte. Josephs Bruder, Maximilian Franz (1756-1801) war seit 1780 Koadjutor des Kölner Erzbischofs gewesen und übernahm dieses Amt nach dessen Tod 1784. Die Urkunde, mit der die „Maxische Akademie“ zu einer Universität wurde, traf wenige Tage nach dem Tod von Maximilian Friedrich in Bonn ein.

Von der Ausrichtung und vom Fächerkanon her entsprach die medizinische Lehre an dieser Universität den Anforderungen einer Medizin, die nicht nur auf das Individuum fokussierte sondern auch die Gesundheitsversorgung der Bevölkerung zum Ziel hatte. Fächer wie Gerichtsmedizin und Medizinische Polizey waren hier von Anfang an vertreten, die Publikationen der Bonner Professoren hatten im Wesentlichen die Verbesserung der Gesundheit der Allgemeinheit und die Förderung des Bevölkerungswachstums zum Gegenstand. Besonders zu erwähnen sind hierbei Arbeiten des Professors für Anatomie und Chirurgie, Joseph Rougemont (1756-1818) über erbliche Krankheiten und zu vom Standpunkt des Mediziners aus gesundheitsschädlichen Kleidungsgewohnheiten von Kindern, Frauen und Männern. ${ }^{10}$ In beiden Publikationen wird ausführlich auf die Anschauungen von Johann Peter Frank Bezug genommen. Auch die Ratschläge für eine gesundheitsfördernde Lebensführung, v. a. die Nutzung von kaltem Wasser, des Arztes und Referenten für das niederösterreichische Sanitätswesen, Johann Pascal Ferro (1753-1809), wurden thematisiert und empfohlen. Franz Gerhard Wegeler (1765-1848), ab 1789 Professor für Gerichtsmedizin und Medizinische Polizey an der Universität Bonn, studierte einige Zeit lang an der medizinisch-chirurgischen Akademie in Wien, mit der die medizinische Fakultät der Universität Bonn offenbar inten-

Josephsakademie in Wien. In: Andreas BERGER (Hg.) Beiträge der Tagung zum 625-jährigen Bestehen der Universität Köln [im Druck].

9 Joseph Claude Rougemont, Abhandlung über die erblichen Krankheiten, übers. von Friedrich Gerhard WEGELER. Frankfurt am Main 1794.

10 Joseph Claude Rougemont, Etwas über Kleidertracht, in wie ferne sie einen nachtheiligen Einfluß auf die Gesundheit hat. Nebst einigen anatomischen und chirurgischen Beobachtungen. Bonn 1784. 
sive Beziehungen pflegte. In diesem Zusammenhang ist noch zu erwähnen, dass nicht nur Fürstbischof Max Friedrich und seine Berater den Ideen des „,aufgeklärten Wohlfahrtsstaates“ zugetan waren, sondern auch sein Nachfolger Maximilian Franz.

Eine ähnliche Parallelität in der medizinischen Denkweise und in der Ausbildung ist auch für Wien nachweisbar. Joseph II. hatte sich einige Zeit lang erfolglos bemüht, die Professoren der Wiener medizinischen Fakultät dafür zu gewinnen, nicht nur eine Medizin zu betreiben und zu lehren, die sich auf das Individuum konzentrierte, sondern auch die Gesundheit der Bevölkerung zu berücksichtigen. Als Resultat dieser gescheiterten Bemühungen wurde 1785 die „Medizinisch-Chirurgische Akademie“ gegründet, die ein Jahr später den Rang einer Universität erhielt und sich nunmehr „Medizinisch-Chirurgische Josephsakademie“ nennen durfte. Schließlich bürgerte sich die Bezeichnung „,osephinum“ ein. Diese Institution stand unter militärischer Verwaltung, was eine direkte Einflussnahme des Kaisers ermöglichte. Allerdings wurden hier nicht nur Ärzte für den militärischen, sondern auch für den zivilen Bereich ausgebildet, die den Anforderungen eines medizinischen Allrounders entsprachen, aber auch in administrativen Belangen und in der Erstellung von Statistiken ausgebildet waren. Weitgehend unbekannt ist, dass am „Josephinum“ auch eine wissenschaftliche Akademie eingerichtet war, die das Ziel verfolgte, medizinisches Wissen zu erweitern und bekannt zu machen. Obwohl ausreichend Quellen vorhanden sind, wurde dieser Tätigkeitsbereich des "Josephinums" noch nicht bearbeitet, diese Akademie ist weitgehend unbekannt. Die Struktur dieser Einrichtung war im Wesentlichen die einer „Akademie der Wissenschaften“ mit Wirklichen und Korrespondierenden Mitgliedern, regelmäßigen Sitzungen, zu denen auch die Protokolle existieren, sowie der jährlichen Ausschreibung von Preisfragen und der Herausgabe einer Zeitschrift. ${ }^{11}$

Die Medizinisch-Chirurgische Akademie war ein kaum zu unterschätzender Teil des Gesundheitswesens im „Josephinismus“, hier wurde vieles umgesetzt, was mit „Ideen der Aufklärung" charakterisiert werden könnte.

Gerade in Bezug auf die gesundheitspolitischen Maßnahmen und diese Institution bestehen derzeit große Forschungsdefizite, zudem hält sich hierfür ein hartnäckiges master narrative. Die Zählebigkeit dieses Narrativs wird durch die allgemeine Annahme unterstützt, dass die Geschichte der Wiener Medizin im 18. und 19. Jahrhundert bereits umfassend erforscht sei. Krassestes Beispiel für dieses Forschungsdefizit ist, dass im Grunde nicht bekannt ist, dass zumindest für einen Zeitraum von etwa einhundert Jahren nach der Gründung der Medizinisch-Chirurgischen Akademie nachgefragt werden müsste, an welcher Institution ein Arzt seine Ausbildung und Graduierung erworben hatte, wenn es heißt, er hätte „in Wien“ studiert. Auch die Ungenauigkeit der Definition dieses Zeitraumes zeigt den enormen Forschungsbedarf in Bezug auf diese Institution, ihre Beiträge zu den medizinischen Wissenschaften und ihre Rolle in der Gesellschaft.

11 Sonia Horn, „... eine Akademie in Absicht der Erweiterung der medizinisch - chirurgischen Wissenschaft ..." Das Josephinum. Hintergründe für die Entstehung der medizinisch-chirurgischen Akademie. In: Renate Zedinger / Wolfgang Schmale (Hg.), Échecs et réussites du Joséphisme/Josephinismus Eine Bilanz. Bochum 2008, 215-244. 
Die „Ausständigkeit“ von Forschungen zu bestimmten Bereichen der Medizin und des Gesundheitswesens des späten 18. Jahrhunderts in den Habsburgischen Ländern und die Betonung anderer Aspekte führt jedoch wieder zum eigentlichen Thema dieses Beitrages zurück, dem „Schreiben über Josephinismus“.

Dass das Josephinum als zentraler Aspekt des „josephinischen“ Gesundheits- und Sozialwesens ebenso wie des Bildungswesens bei Eduard Winter und Fritz Valjavec kaum Berücksichtigung fand, mag weniger überraschen, da in beiden Werken religionsgeschichtliche bzw. kulturgeschichtliche Zugänge vorherrschen. Allerdings wäre es aufgrund der zentralen Bedeutung der Kirche im josephinischen Gesundheits- und Bildungswesen naheliegend gewesen, auch diese Aspekte zu berücksichtigen. Die auffällige Ähnlichkeit der Vorschläge zur Strukturierung des Gesundheitswesens in Winters Habilitation lassen vermuten, dass er hier Anleihen beim josephinischen System genommen haben könnte. Dass dieser Aspekt, das Josephinum als Ausbildungsstätte und „Akademie“, aber auch bei Erna Lesky einen blinden Fleck darstellt, stimmt nachdenklich. Auch die reich bebilderte Publikation über das Josephinum zeigt keine derartigen Bezüge. ${ }^{12}$

Als ,josephinisches“ Gesundheits- und Sozialwesen wurde hauptsächlich die Gründung des Allgemeinen Krankenhauses und der primär humanitäre Hintergrund derselben beschrieben, wobei meist noch die Etablierung des „Unterrichts am Krankenbett“ hier verortet wird - dies ist auch mit einer Gedenktafel am Eingang des heutigen Allgemeinen Krankenhauses dokumentiert. ${ }^{13}$ Letzteres ist freilich, wie sich noch zeigen wird, ein unwiderlegbarer Irrtum, an dem sich auch bei wohlmeinender Interpretation nichts ändert.

Bezüge zum Josephinum finden sich bei Lesky in der Regel lediglich im Zusammenhang mit den anatomischen und geburtshilflichen Wachsmodellen sowie mit der kunsthistorischen Besonderheit, die das Gebäude selbst darstellt. Die Medizinisch-Chirurgische Akademie wird in erster Linie als „militärärztliche“ Ausbildungsstätte dargestellt und somit in einen besonderen Bereich verschoben, der wenig mit dem medizinischen Alltag zu tun hat.

\section{MYSTERIÖSES JOSEPHINUM}

Wie erwähnt, hatte sich Joseph II. darum bemüht, die medizinische Fakultät von einer Ausrichtung der Lehre zu überzeugen, die Gesichtspunkten folgen sollte, die auch für den Staat Relevanz hatten, und war dabei allerdings erfolglos geblieben. Daraus resultierte schließlich die Gründung der Medizinisch-Chirurgischen Akademie, deren Ziel jedoch nicht nur die Ausbildung von Ärzten für den militärischen und den zivilen Bereich war, sondern auch das konsequente Sammeln und Produzieren von relevantem Wissen. Dies kann dahingehend

12 Helmut Wукціску, Das Josephinum: Biographie eines Hauses. Die medizinisch-chirurgische Josephs-Akademie seit 1785; das Institut für Geschichte der Medizin seit 1920. Wien 1985.

13 Helmut LeITNER, Sozialpolitik im aufgeklärten Absolutismus: Kaiser Joseph II und das Spitalswesen in Wien. In: Michael Hubenstorf et al. (Hg.), Medizingeschichte und Gesellschaftskritik. Festschrift für Gerhard BAADER. Husum 1997, 92-100. 
verstanden werden, dass an dieser neuen Institution andere Ausbildungsziele verfolgt wurden als an der medizinischen Fakultät, und dass dies sowohl durch Forschungen als auch durch Publikationen unterstützt wurde. Der Lehrplan für die medizinische Ausbildung unterschied sich von jenem, nach dem an der medizinischen Fakultät gelehrt wurde unter anderem darin, dass Spezialfächer wie Zahnheilkunde, Augenheilkunde, Dermatologie und gerichtliche Medizin im Curriculum vertreten waren, aber auch Arithmetik und Geometrie als Grundlagen der Statistik, die wiederum für die „Staatsarzneikunde“ und „Medizinische Polizey“ benötigt wurde. Der Unterricht folgte anderen didaktischen Konzepten, zudem wurden für die Medizinisch-Chirurgische Akademie eigene Lehrbücher verfasst, vor allem für diese speziellen Fächer.

Dies bedeutet jedoch nicht, dass es zuvor noch keinen Unterricht in einigen Spezialfächern gegeben hätte, die für den Alltag benötigt wurden. So wurde an der medizinischen Fakultät der Universität Wien etwa im Anatomieunterricht von Joseph Barth (1746-1818) auch Augenheilkunde gelesen. Ein Impuls für die Einrichtung der Medizinisch-Praktischen Lehrschule war, dass 1752 in einem Vortrag des Directorium in publicis et cameralibus auf die Notwendigkeit der Einrichtung eines Operationszimmers hingewiesen wurde, in dem unter Anleitung vor allem augenheilkundliche Eingriffe vorgenommen werden sollten. ${ }^{14}$

Damit dürfte auch die 1752 erfolgte Berufung von Guiseppe Natalis Palucci (1719-1797) als Augenarzt zusammenhängen. ${ }^{15}$ Die weitere Entwicklung der Augenheilkunde in dieser Epoche wurde jedoch noch nicht erarbeitet und wurde auch in den rezenten Feiern zum zweihundertjährigen Jubiläum der Gründung der Wiener Augenklinik, die als weltweit erste propagiert wird, nicht berücksichtigt, ebenso wenig wie die Tatsache, dass die Augenheilkunde als eigenes Fach ab 1785 an der Medizinisch-Chirurgischen Akademie unterrichtet wurde.

Die Definition eines Unterrichtsfaches mit einem hierfür angestellten Lehrenden und speziellen Lehrbüchern, dies zudem im Rahmen eines eigenen Lehrplanes, ist jedoch ein Schritt zur Spezialisierung und Teil der Etablierung eines medizinischen Faches. In diesen Fällen ging es zudem um die Berücksichtigung von medizinischem Wissen, das für die alltägliche Praxis und die Allgemeinheit gebraucht wurde. Am besten kann dies anhand der Vorrede der Materia Chirurgica von Joseph Jakob Plenk (1735-1807) gezeigt werden. ${ }^{16}$ Es ging dem Autor darum, das „von alters her" bekannte und umfassende Wissen der Bader und Wundärzte über die in erster Linie konservativen Behandlungen von Erkrankungen aufzuschreiben, damit dieses Wissen an nachfolgende Generationen weitergegeben werden konnte. In gewisser Weise erinnert dies auch an das ebenfalls im 18. Jahrhundert einsetzende Interesse an münd-

14 Daniela WAGNer, Gérard van Swieten und die Gründung der Kliniken in Wien. Eine verwaltungsgeschichtliche Analyse auf der Basis archivalischer Quellen. Masterarbeit Universität Wien 2015, 9 , $27-29$.

15 Wagner, Gründung der Kliniken, wie Anm. 14, 10; sowie Erna Lesky, Gerhard van Swieten. Auftrag und Erfüllung. In: EAD. / Adam WANDruszKa (Hg.), Gerhard van Swieten und seine Zeit. Wien 1973, 11-61, hier 28 [Anm. 71].

16 Joseph Jacob Plenk, Materia chirurgica, oder Lehre von den Wirkungen der in der Wundarzney gebräuchlichen Heilmittel. Wien ${ }^{2} 1777$. 
lich tradierten Sprach- und Rechtsdenkmälern. Von Interesse ist weiters, dass auch auf die Ähnlichkeit einheimischer Therapieformen mit jenen anderer Regionen Bezug genommen wird, etwa wenn von der Moxatherapie berichtet wird: „Die Manier, wie die Japaneser die Moxa zubereiten, scheinet in allen Stücken mit der übereinzukommen, wie wir sie von unserm einheimischen Beifuße erhalten." 17

Die Lehrbücher von Joseph Plenk waren aufgrund ihrer übersichtlichen Strukturierung und wohl auch wegen ihrer leicht fasslichen Darstellungsweise weit verbreitet. Sie wurden meist in Deutsch verfasst und in zahlreiche Sprachen übersetzt. Dass die Zielgruppe der medizinischen Ausbildung am Josephinum Ärzte waren, die dem Staat nützlich sein und zum Einsatz für das Wohl der Allgemeinheit ausgebildet werden sollten, findet sich laufend in den für diese Institution verfassten Lehrbüchern, nicht nur in jenen von Joseph Plenk. Dies wurde vor allem in den Paratexten klar angesprochen, findet sich jedoch auch im Text und in den vermittelten Inhalten selbst. ${ }^{18}$

Diesen Publikationen ist jedoch auch zu entnehmen, dass zwischen den am Josephinum und an der medizinischen Fakultät vertretenen Lehrmeinungen ebenso Unterschiede bestanden wie zwischen einigen der propagierten therapeutischen Vorgehensweisen. Besonders deutlich wird dies bei der Haltung gegenüber der Pockenimpfung. Wohl aufgrund persönlicher Erfahrungen waren Maria Theresia und Joseph II. von der Sinnhaftigkeit dieser Maßnahme überzeugt, ebenso wie die am Josephinum tätigen Ärzte. Gérard van Swieten hingegen war der Impfung eher skeptisch gegenübergestanden, sein Kollege Anton de Haen (1704-1776), immerhin Leiter der Medizinisch-Praktischen Lehrschule der medizinischen Fakultät, polemisierte heftig gegen diese Maßnahme. ${ }^{19}$

Die Aufnahme von Studenten erfolgte an der Medizinisch-Chirurgischen Akademie durch ein Verfahren, das auch jenen die Möglichkeit zu studieren gab, die weder über den hierfür nötigen sozialen Hintergrund noch über ein ausreichendes Vermögen verfügten. Dies entspricht den politischen Theorien von Joseph von Sonnenfels (1732/33-1817), der unter anderem forderte, dass nicht nur Kinder aus begüterten Familien Zugang zu einer höheren Bildung haben sollten. Begabung und Intellekt waren seiner Meinung nach nicht vom Elternhaus abhängig, vielmehr sollte gezielt nach begabten jungen Menschen gesucht werden, damit diese nicht nur zu tüchtigen Staatsbürgern, sondern auch zu Akademikern ausgebildet werden konnten, die dem Wohl der Allgemeinheit dienen und auch im staatlichen Dienst eingesetzt werden konnten. ${ }^{20}$ Tatsächlich sind bereits einige Biographien bekannt, für die nachgewiesen werden konnte, dass die Ausbildung am Josephinum soziale Mobilität nach

17 HütTer, Materia Chirurgica, wie Anm. 7, 83.

18 HÜtтеR, Materia Chirurgica, wie Anm. 7, 30-36. Hier wird besonders das Kapitel Die ächte Weise dem Staate nützliche Wundärzte zu bilden in: Joseph Jakob Plenk, Sammlung von Beobachtungen über einige Gegenstände der Wundarzneywissenschaften. 2 Bde., Wien 1769-1770, thematisiert.

19 Ausführich dazu: Norman Beale / Elaine Beale, Echoes of Ingen Houz. The Long Lost Story of the Genius Who Rescued the Habsburgs from Smallpox and Became the Father of Photosynthesis. East Knoyle 2011.

20 Sonia Horn, Entstehung der medizinisch-chirurgischen Akademie, wie Anm. 11, 238. 
oben sowohl für den Betreffenden als auch für seine Nachkommen mit sich brachte. ${ }^{21}$

Die Medizinisch-Chirurgische Akademie ist somit als „politischer Ort“" zu verstehen. Hier wurden medizinische Inhalte vermittelt, die auch den wirtschafts- und gesellschaftspolitischen Zielsetzungen von Joseph II. und seinem Kreis entsprachen. Dasselbe gilt für die praktische Umsetzung von bildungspolitischen Konzepten.

Gerade die gesellschaftspolitischen Vorstellungen Josephs, ebenso wie die damit verbundenen Bildungskonzepte, waren freilich im ausgehenden 18. Jahrhundert, besonders im Vormärz, umstritten, wurden aber dennoch in gewisser Weise weiter geführt. ${ }^{22}$ Ihre (freilich mehrschichtige und komplexe) Bedeutung im Zusammenhang mit den Geschehnissen von 1848 ist historiographisch dokumentiert, und auch auf eine verhältnismäßig hohe Beteiligung von Studenten der Medizin an den revolutionären Ausschreitungen 1848 wurde bereits hingewiesen. ${ }^{23}$ Nicht hinterfragt wurde bisher jedoch, ob diese Medizinstudenten an der medizinischen Fakultät oder am Josephinum studierten.

Bemerkenswert ist weiters die Tatsache, dass die verschiedenen in Italienisch gehaltenen öffentlichen Vorträge des ersten Direktors der Medizinisch-Chirurgischen Akademie, Giovanni Alessandro Brambilla (1728-1800), im Original wesentlich diplomatischer formuliert waren, als dies die publizierten deutschen Übersetzungen vermuten lassen. In diesen erscheint Brambilla als scharfer Kritiker der bestehenden Medizin und der bestehenden medizinischen Ausbildung. ${ }^{24}$ Brambilla selbst dürfte nach dem Tod von Joseph II. aus politischen Gründen von seinem Posten abberufen worden sein. Dass die Medizinisch-Chirurgische Akademie eine an sich umstrittene Institution war, zeigen unter anderem die sarkastischen Beschreibungen der „flachbäuchigen Eleven“ dieser Institution und die Kritik an den im Garten für jeden zugänglich ausgestellten Präparaten. ${ }^{25}$

Ging es in der Folge also darum, diese politischen Ziele und die damit verbundene Institution aus der medizinischen Geschichtsschreibung ,auszublenden“? Warum ist der Aspekt „Gesundheits- und Sozialwesen“ meist auf einen historisch unzutreffenden Lobgesang auf die humanitäre Gesinnung von Joseph II. reduziert, aus der auch die Gründung des Allgemeinen Krankenhauses abgeleitet wurde? Ist deswegen das Gesundheits-, Sozial- und Bildungswesens in der Geschichtsschreibung des 19. und frühen 20. Jahrhunderts weitgehend unberücksichtigt geblieben, auch im Verständnis von „Josephinismus“ bei Eduard Winter und Fritz Valjavec?

21 Rudolf RaBL, Anfänge, Ausbreitung und Werdegang der Ärztefamilie Rabl in Oberösterreich. In: Jahrbuch des Oberösterreichischen Musealvereins 115 (1970), 167-198.

22 Vgl. dazu Franz Leander Fillafer, Die Aufklärung in der Habsburgermonarchie und ihr Erbe. Ein Forschungsüberblick. In: Zeitschrift für Historische Forschung 40/1 (2013), 35-97.

23 Katharine Elisabeth Kogler, ,... die Heilärzte des kranken Staates“. Die Beteiligung von Medizinern an der Revolution 1848 in Wien. Wien 2012.

24 Barbara Peintinger, Giovanni Alessandro Brambillas Appendice: Eine Quelle zur Geschichte des Gesundheitswesens im Josephinismus. Diplomarbeit Universität Wien 2010, 44, $57 \mathrm{f}$.

25 Anna Märker, Model Experts: Wax Anatomies and Enlightenment in Florence and Vienna, 17751815. Manchester 2011, 159-165. 


\section{IV „KLIO UND HIPPOKRATES“ ${ }^{26}$}

Naheliegend ist der Versuch, diese Frage zunächst anhand der Geschichte der Wiener Medizingeschichtsschreibung zu klären.

Medizingeschichte ist ein Lehr- und Forschungsgegenstand, der sich im Lauf des 18. Jahrhunderts aus dem Anspruch und der Notwenigkeit ergab, die meist lateinischen Texte älterer Autoren verständlich zu machen. Über eine inhaltliche Aufbereitung im Rahmen der medizinischen Lehre sollten Studenten das bestehende Wissen kennenlernen, auch durch die Übersetzung von medizinischen Werken sollte dieses Ziel verfolgt werden. Zielpublikum hierbei waren aber nicht nur die Studierenden, denn auch die philosophische Auseinandersetzung mit dem, was Medizin an sich ist oder sein sollte, spielte bei der Verbindung von „Klio und Hippokrates" eine Rolle.

Für die habsburgischen Länder kam durch die 1781 erfolgte Einführung von Landessprachen als Unterrichtssprachen an einigen Universitäten auch der Bedarf an Übersetzungen von lateinischen Texten v. a. ins Deutsche hinzu. Ein weiterer Aspekt war der methodologische Ansatz, dem sich die medizinische Forschung dieser Zeit verpflichtet fühlte, nämlich - mit dem ihr zugrundeliegenden hippokratisch-galenischen Konzept - möglichst viele Erfahrungen zusammenzutragen und diese an die scientific community weiterzugeben. Die intensive wissenschaftliche Kommunikation innerhalb der „Gelehrtenrepublik“ wurde als wesentlicher Prozess in der Weiterentwicklung von medizinischem Wissen verstanden. Mit dieser Vorstellung einer „Gelehrtenrepublik“, die, unabhängig von politischen Vorgaben, anderen Beschränkungen des Wissensaustausches und Sprachunterschieden, über wissenschaftliche Themen kommunizierte, war durchaus auch der Anspruch verbunden, dass die Ergebnisse „unbeeinflusst“ gewonnen und zur allgemeinen „Vermehrung des Wissens“ weitergegeben werden sollten. Jahresberichte aus verschiedenen Krankenhäusern waren neben den entstehenden wissenschaftlichen Fachjournalen hierfür typische literarische Genres. Es ist nachvollziehbar, dass diese Berichte nicht unbedingt von einem ausgelasteten medizinischen Leiter eines Krankenhauses oder einem in der Lehre engagierten Professor verfasst werden konnten. Daher wurde dies von meist medizinisch ausgebildeten Personen übernommen, die über die nötige literarische Kompetenz, aber auch über Sprachkenntnisse verfügten. Sie vertraten dann einen Bereich, der als „medizinische Literatur und Geschichte“ bezeichnet wurde. ${ }^{27}$

Soweit bekannt, war Joseph Eyerel der erste mit einem konkreten Lehrauftrag und mit einer Besoldung ausgestattete Vertreter dieser Disziplin in Wien. ${ }^{28}$ Allerdings ist über ihn

26 Hans-Uwe Lammel, Klio und Hippokrates. Eine Liaison littéraire des 18. Jahrhunderts und ihre Folgen für die Wissenschaftskultur bis 1850 in Deutschland. Stuttgart 2005.

27 Ein gutes Beispiel hierfür sind die von Eyerel herausgegebenen Berichte aus der Wiener Medizinisch-Praktischen Lehrschule sowie die ebenfalls von Eyerel herausgegeben Vorlesungen von Anton de Haen und Maximilian Stoll.

28 Universitätsarchiv Wien [UAW], Currentakten [CA], 1.2.332. (11. April 1809): „Die Niederösterreichische Regierung teilt dem Konsistorium mit, daß dem Dr. Eyerel gestattet wurde, außerordentliche 
bislang kaum Biographisches bekannt. ${ }^{29}$ Er ist zudem der Autor einiger Übersetzungen antiker Autoren ins Deutsche, etwa der Beschreibung der Germanen von Tacitus ${ }^{30}$ oder der Darstellung der attischen Pest von Thukydides, die er durch einen zeitgenössischen Bericht über eine Seuche, die während der napoleonischen Feldzüge Ägypten erfasst hatte, ergänzte. ${ }^{31}$ Eyerel übersetzte auch zeitgenössische Texte aus verschiedenen Sprachen ins Deutsche und veröffentlichte zudem eine Beschreibung der österreichischen Expedition auf die Nikobaren..$^{32}$ Auch eine Zusammenarbeit mit dem „Begründer der deutschen Medizingeschichtsschreibung", Kurt Spengel, dürfte stattgefunden haben.

Des Weiteren wurden von Eyerel die Vorlesungen von Anton de Haen und Maximilian Stoll (1742-1787) veröffentlicht, ebenso die jährlichen Berichte aus der Medizinisch-Praktischen Lehrschule in Wien. Er fungierte auch als Herausgeber einer medizinischen Fachzeitschrift, Medizinische Chronik, in der neben Berichten über Therapiemethoden unter anderem durchaus auch sehr freimütig über den aktuellen Zustand der medizinischen Lehre in Wien diskutiert wurde. In dieser Zeitschrift finden sich auch einige rein medizinhistorische Beiträge.

Der Antrag, Eyerel als außerordentlichen Professor für „Medizinische Literatur und Geschichte" mit Besoldung anzustellen, enthielt auch den Hinweis darauf, dass dieser bereits lange Jahre hindurch in diesem Fach erfolgreich tätig gewesen war. Berücksichtigt man seine Publikationstätigkeit, ist dies durchaus nachvollziehbar. Eyerel wurde schließlich mit 11. 4. 1809 zum außerordentlichen Professor für "Medizinische Literatur und Geschichte“ mit Besoldung ernannt. Aufgrund des aktuellen Fehlens von biographischen Daten kann heute jedoch nicht nachvollzogen werden, wie lange Eyerel in dieser Funktion tätig war.

Auch hier drängt sich die Frage auf, warum selbst unter Fachleuten der Medizingeschichte bislang nicht bekannt ist, dass es Ende des 18. Jahrhunderts bzw. definitiv seit 1809 in Wien einen „ersten“ Professor für Medizingeschichte gab, auch wenn die Bezeichnung geringfügig

Vorlesungen über medizinische Literatur zu halten. Die Vorlesung soll an der Universität gehalten werden. Das Honorar soll von Dr. Eyerel festgesetzt werden; Stipendiaten und Studenten, die vom Unterrichtsgeld befreit sind, sollen die Vorlesung unentgeltlich besuchen dürfen. Die Vorlesung darf solange gehalten werden, bis die Erlaubnis von der Regierung und vom Studiendirektor widerrufen wird."

29 Lesky erwähnt als frühe Medizinhistoriker auch Ludwig von Attenhofer und Andreas Ignaz Wawruch, die ihrer Meinung nach jedoch kaum von Bedeutung waren: Erna Lesky, Die Wiener medizinische Schule im 19. Jahrhundert. Graz - Wien 1965, 617-620.

30 Joseph Eyerel (Hg.), Tacitus' Germanien. Übersetzt mit Erläuterungen von Kurt Sprengel. Wien 1819.

31 Joseph Eyerel, Von der Pest in Athen. Aus dem Griechischen übersetzt, mit Zusätzen und Anmerkungen, und mit einem Anhang über die Pest in Egypten, während den französischen Feldzügen von Doctor Paul Assalini (Ein Auszug aus dem Protocoll der medizinischen Schule von Paris) von Doctor Joseph Eyerel, ausserordentlichem Professor der medizinischen Geschichte und Literatur. Wien 1810.

32 Joseph Eyerel (Hg.), Johann Friedrich Blumenbach's Anfangsgründe der Physiologie, aus dem Lateinischen übersetzt und mit Zusätzen vermehrt von Joseph Eyerel. Wien ${ }^{2} 1795$; ID. (Hg.), Tagebuch der Reise des kais. kön. Schiffes Joseph und Theresia nach den neuen österreichischen Pflanzorten in Asia und Afrika. Von Nikolaus Fontana, gewesenen Schiffwundarzt, an Herrn Brambilla, Leibwundarzt Sr. Mt. des Kaisers und Protochirurgus der k. Armeen. Dessau - Leipzig 1782. 
anders war. Erna Lesky erwähnt lediglich, dass Eyerel medizinhistorische Vorlesungen gehalten habe. ${ }^{33}$ Karl Holubar erklärte in einem 1990 erschienen Beitrag in der Wiener medizinischen Wochenschrift detailliert und ausführlich, warum als Gründungsdatum der Wiener Medizingeschichte der 20. August 1848 anzunehmen wäre, das Datum also, das der Bescheid trägt, mit dem Romeo Seligmann (1808-1892) ${ }^{34}$ zum außerordentlicher Professor für Medizingeschichte ohne Besoldung ernannt wurde; und nicht, wie Erna Lesky ausgeführt hatte, der 3. Dezember 1850, der Tag, an dem Seligmann schließlich auch eine Besoldung für seine Tätigkeit erhielt. ${ }^{35}$ Zieht man in Betracht, dass es heute nicht schwierig ist und auch vor mehreren Jahrzehnten nicht schwierig war, zu eruieren, dass Eyerel 1809 eine immerhin bezahlte Professur in diesem Fach erhalten hatte, stellt sich mit einiger Dringlichkeit die Frage, warum auf ihn nicht eingehender Bezug genommen wurde, auch nicht von früheren Medizinhistorikern wie Romeo Seligmann, dem Eyerel durchaus bekannt gewesen sein könnte.

Diese „Positionierung“ Eyerels ist auch im Hinblick auf die selektive Wahrnehmung des Josephinismus aussagekräftig. Eyerel vertritt in manchen Bereichen eine Meinung, die jener der Vertreter der medizinischen Fakultät widerspricht - etwa in der Frage der Pockenimpfung. Er diskutiert und befürwortet diese in seinem Text Praktische Beyträge zur Geschichte der Kinderpocken und der Kuhpocken ${ }^{36}$ und stellt sich somit gegen die auf Anton de Haen zurückgehende Anschauung. Aufgrund der persönlichen Erfahrungen von Joseph II. mit den Pocken und der Meinung der Ärzte aus seinem Kreis ist nachvollziehbar, dass man am Josephinum die Pockenimpfung nicht nur befürwortete, sondern dass auch die Notwendigkeit einer Durchimpfung der gesamten Bevölkerung vertreten wurde. Dies wurde im Rahmen der „josephinischen“ Reformen im Gesundheitswesen auch umgesetzt, indem die flächendeckende Impfung über die Pfarren organisiert wurde. Eyerel widmete (zumindest) ein Buch Joseph Plenk, dem bedeutenden Lehrer am Josephinum. Außerdem vertrat Eyerel in Bezug auf die Entwicklung des Gesundheitswesens Positionen, die ihn in die Nähe jener Meinungen rückt, die der Medizinisch-Chirurgischen Akademie zugeordnet werden können.

Könnte die „Nichtpräsenz“ dieses frühen Fachvertreters in der späteren Medizingeschichtsschreibung ein Hinweis auf eine Art damnatio memoriae von "Josephinischem“ im Zusammenhang mit der Medizinisch-Chirurgischen Akademie sein?

Ein weiteres Indiz hierfür drängt sich auch im Fall des Wiener Medizinhistorikers (und

33 LESKY, Die Wiener medizinische Schule im 19. Jahrhundert, wie Anm. 29, $618 f$.

34 Franz Romeo Seligman studierte Medizin und orientalische Sprachen in Wien und war nach seiner Promotion zum Doktor der Medizin als Cholera-Arzt im Einsatz. Er widmete sich besonders der Geschichte und Orientalistik. 1848 war er zunächst unbesoldet, ab 1850 besoldet als Professor für Medizingeschichte tätig.

35 Karl Holubar, Zur Vorgeschichte der Gründung des medizinhistorischen Lehrstuhles an der Universität Wien (1833 bis 1848/1850). In: Wiener Medizinische Wochenschrift 140/17 (15. 9. 1990), 441445.

36 Joseph Eyerel, Praktische Beyträge zur Geschichte der Kinderpocken und der Kuhpocken. Wien 1800. Eyerel initiierte auch die Herausgabe einer Zeitschrift, in der die Erfahrungen mit der Kuhpockenimpfung publiziert werden sollten, die Annalen der Kuhpockenimpfung. Diese dürfte jedoch nicht über den 1802 publizierten ersten Band hinausgegangen sein. 
eben nicht ersten Professors für Medizingeschichte), Romeo Seligmann, auf. Seligmann hatte sich spätestens seit 1833, dem Jahr der anzunehmenden Pensionierung von Eyerel, darum bemüht, eine Professur für Medizingeschichte zu erhalten. Allerdings wurden die Gesuche immer wieder abgelehnt, obwohl er kontinuierlich Lehrveranstaltungen hielt und auch eine beeindruckende Übersetzung des Textes einer persischen Handschrift, der Grundsätze der Pharmakologie, die im zehnten Jahrhundert Abu Mansur Muvaffak Harawi verfasst hatte. ${ }^{37}$ An Seligmanns Qualifikation für das Fach dürfte diese lange Wartezeit nicht gelegen sein. Finanzielle Gründe waren jedoch häufig die Ursachen für die Ablehnung von derartigen Ansuchen. Seine beiden Brüder Franz und Leopold hatten ihre medizinischen Ausbildungen zum Teil am Josephinum absolviert, Franz Seligmann (1808-1889) nahm als leitender Arzt an der Novara-Expedition, der österreichischen Weltumsegelung der Jahre 1857-1859, teil. ${ }^{38}$ Seligmann stammte, wie Joseph von Sonnenfels, aus einer Nikolsburger jüdischen Familie und konvertierte zum katholischen Glauben. Auch er besuchte das Nikolsburger Piaristengymnasium und interessiertes sich für Literatur und orientalische Sprachen. In einer Festrede vor dem Doktorenkollegium der Wiener medizinischen Fakultät deklarierte sich Seligmann 1861 ziemlich eindeutig:

Dazu aber bedarf es nicht nur der geistigen Arbeit der einzelnen Berufenen, dazu bedarf es der ernsten, ja grossartigen Mitwirkung des Staates selbst, im vorurtheilsfreien, grandiosen - im Josephinischen Style. ${ }^{39}$

Vielleicht waren diese Faktoren dafür mitverantwortlich, dass er sehr lange auf seine Ernennung warten musste und diese erst im Revolutionsjahr 1848 erfolgte.

Auffällig ist jedoch, dass Seligmann zumindest nach seiner Ernennung zum Professor in seinen Publikationen, vor allem in seinen überlieferten Vorträgen, die Medizinisch-Chirurgische Akademie weitgehend unberücksichtigt ließ, aber die aktuellen Errungenschaften seiner Kollegen stark, nachgerade polemisch, ins Licht rückte. In seinen Texten finden sich schließlich auch die Bezeichnungen „erste und zweite Wiener Medizinische Schule“, die bis heute stark wertungsbehaftete ,aufgeladene“ Begrifflichkeiten sind. ${ }^{40}$ Der Blick vor die Zeiten von Gérard van Swieten hat in dieser Art der Medizingeschichtsschreibung keinen Platz, Verweise auf diese Epoche dienen lediglich dem Kontrasteffekt, sie wird als vorwissenschaftliches, dunkles Zeitalter der Spekulation verstanden:

37 Romeo Seligmann (Hg.), Liber fundamentorum prarmacologiae. Codex Vindobonensis sive medici Abu Mansur Muwaffak Bin Ali Heratensis Liber fundamentorum pharmacologiae linguae ac scripturae Persicae specimen antiquissimum. 2 Bde., Wien 1830-1833.

38 Daniela Angetter, Art. Seligmann, Franz Romeo. In: Neue Deutsche Biographie 24 (2010), 221-222.

39 Franz Romeo Seligmann, Adam Chenot und seine Zeit. Wien 1861, 4. - Zu Seligmann und der Rolle der Mediziner in der Revolution von 1848: Katharina Kogler, „... die Heilärzte des kranken Staates.“ Die Beteiligung von Medizinern an der Revolution von 1848. Wien 2012.

40 Franz Romeo Seligmann, Die Heilsysteme und die Volkskrankheiten. Eine Vorrede. Wien 1850, 13. Vgl. dazu auch Seligmann, Festrede, wie Anm. 39. 
Nach überlanger Nacht brach der Lichtstrahl aus der Ferne mächtig herein; denn spät traten Österreichs Ärzte in die Reihe der medicinischen Größen ersten Ranges. ${ }^{41}$

Maria Theresia, van Swieten und andere Größen der ersten Wiener Schule werden in folgender Weise dargestellt:

Mit Maria Theresia begann die Epoche des höchsten Glanzes. Der grösste Schüler des grossen Meisters: Van Swieten, dessen edler Genius das gesammte medicinische Wissen umfasste und verschwenderisch liebevoll ausstreute, der unduldsame selbst fanatische aber in seinem Kreise klare ja große de Haen, - Stoll der stille, bescheidene, unsterbliche, - Auenbrugger der Virtuose auf dem Thorax, und viele Andere, schufen der ersten Wiener Schule einen nie verlöschenden Ruhm. ${ }^{42}$

Über die Entstehung der zweiten Wiener Schule wird berichtet:

Die zweite Wiener Schule entstand, eine Tochter der Fremde wie die Erste; wie diese nach langem Stillstande, von Decennien zwar, aber Decennien sind was Jahrhunderte waren. Die unermesslichen Schätze des Wiener Krankenhauses schliefen im strengsten Sinne des Wortes den Todesschlaf, Rokitansky weckte sie; den Tausenden von Leichen gab er ein neues wissenschaftliches Leben. ${ }^{43}$

Es stellt sich somit die Frage nach dem Grund einer solchen Darstellung und der Konstruktion einer Datumsgrenze in der Mitte des 18. Jahrhunderts, die an das „Erscheinen“ der Heldenfigur Gérard van Swieten gebunden wurde. Dies ging mit der Ausklammerung einiger Jahrhunderte vor dieser Epochenschwelle einher und auch mit der unzweideutigen Charakterisierung der Medizin vor Gérard van Swieten als „spekulativ“. Die Etablierung dieses master narrative erfolgte zudem durch Romeo Seligmann, einen Wissenschafter, der keinerlei Probleme bei der Heranziehung lateinischer Quellen gehabt hätte, wie etwa der seit 1399 überlieferten Akten der Wiener Medizinischen Fakultät, und aus diesen ganz andere Sachverhalte berichten hätte können. Weiters ist davon auszugehen, dass Seligmann die Inhalte dieser „spekulativen“ Medizin bekannt waren, zumal dieses Lehrgebäude während seiner medizinischen Ausbildung noch relevant war und anzunehmen ist, dass ihm die diesbezügliche Literatur zugänglich - auch im Sinne von verständlich - war.

In die Reihe von derartigen Darstellungen der Geschichte der Medizin in Wien fügt sich auch die 1843 von Anton von Rosas (1791-1855) veröffentlichte Geschichte der Universität Wien im Allgemeinen und der medizinischen Fakultät im Besonderen ${ }^{44}$ ein. Die in vielen Be-

41 Seligmann, Heilsysteme, wie Anm. 40, 13.

42 Seligmann, Heilsysteme, wie Anm. 40, 14.

43 Seligmann, Heilsysteme, wie Anm. 40, 15.

44 Anton Rosas, Kurzgefasste Geschichte der Wiener Hochschule im Allgemeinen und der medicinischen Facultät derselben insbesondere. Wien 1843. 
reichen verfälschende Wiedergabe der Quellen beruht meist auf paläographischen Mängeln und mangelhaften Lateinkenntnissen, worauf auch Karl Schrauf in der Einleitung zu seiner Edition der Akten der Wiener Medizinischen Fakultät hingewiesen hat. ${ }^{45} \mathrm{Zu}$ erwähnen ist außerdem die auffällig antisemitische Auslegung einiger Textstellen, die mit Einstellungen einhergehen, die sich auch anderen seiner Publikationen entnehmen lassen. ${ }^{46} \mathrm{Ob}$ dies als Verzerrung angelegt war, sei dahingestellt, jedenfalls leistete diese Darstellung einer Interpretation der Jahrhunderte vor 1750 als einer einigermaßen dunklen Epochen von ,spekulativer" Medizin Vorschub. ${ }^{47}$ Welche Motivationen konnten aber, mehr oder weniger bewusst intendiert, hinter einer solchen Perspektivierung stehen?

Eine andere Spur im Hinblick auf die Nicht-Wahrnehmung des Josephinums führt in gesundheits- und hochschulpolitische Bereiche. 1794 hatte Andreas von Stifft (1760-1836) für die Beantwortung der Preisfrage, wie man die Medizinisch-Chirurgische Josephsakademie zweckmäßig reorganisieren könne, einen Preis von 40 Dukaten erhalten. ${ }^{48}$ Aufgrund dieser Ausführungen wurde der Protomedikus Anton von Störk (1731-1803) auf ihn aufmerksam, was schließlich dazu führte, dass Stifft 1803 dessen Nachfolge antrat. Gleichzeitig war er aber auch Direktor der medizinischen Studien an der Universität Wien, Präses der Medizinischen Fakultät, Bücherzensor für medizinische Literatur und persönlicher Arzt des Kaisers. Diese Information ist seinen Nachrufen zu entnehmen, archivalische Quellen zu dieser Preisfrage und den konkreten Vorschlägen wurden bislang jedoch noch nicht aufgefunden. Bei dem genannten Preisgeld handelt es sich um den üblichen Betrag, der vom Josephinum für Preisfragen ausgelobt wurde, was die Annahme nahelegt, dass diese Frage von ebendieser Institution aufgestellt wurde. Erna Lesky erwähnt noch weitere Ehrungen und Mitgliedschaften in wissenschaftlichen Akademien, was sie als eine Machtfülle schildert, über die zuvor nur Gérard van Swieten verfügt hatte.

Alles wie van Swieten, alle Ämter, alle Würden, alle Titel kehren bei ihm [Stifft] wieder. Aber welcher Mann trug sie nun! Die staatsrätliche Exzellenz hat nie gerne von ihrer Herkunft gesprochen. Vermutlich erinnerte sich Stifft nicht gerne daran, dass er der Sohn bürgerlicher Eltern aus Röschitz in Niederösterreich war. Dort wurde er nämlich geboren,

45 Karl Schrauf (Hg.), Acta Facultatis Medicae Universitatis Vindobonensis 1399-1435. Wien 1899, X.

46 Anton Rosas, Über die Quellen des heutigen ärztlichen Missbehagens und die Mittel um denselben wirksam zu steuern. In: Medizinische Jahrbücher des Kais.-Königl. Österreichischen Staates 40 (1842), 1-19. Als Gründe des Missbehagens bringt Rosas unter anderem vor: „8. Zu grosser Andrang der Israeliten zur Medicin“ (2); „Die überhand nehmende Zahl israelitischer Ärzte gereicht der Medizin als Kunst und Wissenschaft ja selbst der Menschheit zum Nachteil.“ (16).

47 Ausführlich: Sonia Horn, Approbiert und examiniert. Die Wiener medizinische Fakultät und nicht-akademische Heilkundige in Spätmittelalter und früher Neuzeit. Dissertation Universität Wien 2001, 24 [Anmerkung 47], 35, 74-76, 208-212.

48 Johann Jacob SACHS, Medizinischer Almanach für das Jahr 1838 (1838), 34-36; Nekrolog Andreas von Stifft. In: Der Telegraph, österreichisches Conversationsblatt 78 (27. 6. 1838) 309-310; sowie Gabriela Sснмidт-Wyкuitzky, Art. Stifft, Andreas Joseph Frh. von (1760-1836), Mediziner. In: Österreichischen Biographisches Lexikon 1815-1950, 13. Wien 2009, 257-258. 
studierte dann in Wien bei Stoll Medizin und machte sich dessen humoral-pathologische Lehren so zu eigen, daß sie ihm ein ganzes Leben unverrückbare Doktrin blieben. Sein Ehrgeiz begnügte sich nach seiner Promotion 1784 nicht damit, praktischer Arzt zu sein. Man mußte literarisch hervor treten wie einst der Protomedicus Störck und tat dies am besten, dem Vorbilde nacheifernd, auf dem Gebiete der Therapie. ${ }^{49}$

Stiffts preisgekrönte Vorschläge zur Reorganisation der Medizinisch-Chirurgischen Akademie werden bei Lesky nicht erwähnt, in ihren Darstellungen wird er als „Reaktionär“ bezeichnet und als jener Akteur beschrieben, der bereits zu Beginn des 19. Jahrhunderts die „Professorenverfolgung“, die nach den Karlsbader Beschlüssen von 1819 einsetzte, im medizinischen Bereich begonnen hatte. ${ }^{50}$ Auf Stifft soll zudem auch die Etablierung einzelner Fächer wie der Staatsarzneykunde und der Augenheilkunde an der Medizinischen Fakultät zurückgehen, indem diese einerseits als verpflichtende Fächer im Curriculum eingeführt, andererseits auch Lehrstühle und Kliniken eingerichtet wurden. Zur Ausbildung von Chirurgen wurde am Allgemeinen Krankenhaus ein „Operateurinstitut“ gegründet, für das die Ausbildung an der Medizinisch-Chirurgischen Akademie als Vorbild gedient haben soll. ${ }^{51}$

Es liegt nahe anzunehmen, dass Stifft, der die Charakteristika der Medizinisch-Chirurgischen Akademie kannte, dasjenige an die medizinische Fakultät übernahm, was schon einige Zeit zuvor am Josephinum üblich war und sich dort wohl bewährt hatte. Wenn Stifft also als ein wesentlicher Vertreter der Restauration zu sehen ist, kann daraus geschlossen werden, dass die Medizinisch-Chirurgische Akademie nach wie vor als "politischer Ort" wahrgenommen wurde, zumal dort einiges umgesetzt worden war, das man als ,aufklärerisch“ oder ,josephinisch“ bezeichnen könnte; und dass die Frage der Weiterführung einer solchen Institution durchaus kontrovers gesehen wurde. Zwischen 1822 und 1824 fanden jedenfalls an der Medizinisch-Chirurgischen Akademie kaum Lehrveranstaltungen statt, in diesen Jahren wurden auch keine neuen Studenten aufgenommen. Offenbar wurde nunmehr Bewährtes an die Medizinische Fakultät übernommen - allerdings ohne die zu Grunde liegenden gesellschaftspolitischen Konzepte; vielleicht glückt hier aber zugleich auch das organisatorische Durchgreifen des Staates auf die universitäre Medizin. Claudia Wiesemann hat in ihrer elaborierten Studie zum therapeutischen Nihilismus von Joseph Dietl auf die vielfältigen Interessen der ärztlichen Standespolitik und der Universität im Vormärz hingewiesen, ebenso wie auf die zeitgenössische Kritik an den beiden Größen der Wiener Medizin dieser Epoche, Carl von Rokitansky (1804-1878) und Joseph Skoda (1805-1881). ${ }^{52}$ Skoda wurde

49 Lesky, Österreichisches Gesundheitswesen, wie Anm. 3, 216.

50 LESKY, Österreichisches Gesundheitswesen, wie Anm. 3, 219; ausführlich: EAD., Die Wiener medizinische Schule, wie Anm. 29, 32-39.

51 Gabriela Sснміdт-WyкLiтzкy, Art. Stifft, wie Anm. 48, 257.

52 Claudia Wiesemann, Josef Dietl und der therapeutische Nihilismus. Zum historischen und politischen Hintergrund einer medizinischen These. Frankfurt am Main 1991; EAD., Der Aufstand in der Fakultät. Zur rhetorischen Funktion des „therapeutischen Nihilismus“ im vormärzlichen Wien. In: History and Philosophy of the Life Sciences 15/2 (1993), 181-204. 
von zahlreichen Kollegen wegen seines Nicht-Therapierens von Patientinnen und Patienten, die er im Allgemeinen Krankenhaus betreute, angegriffen. Er folgte der Anschauung, dass die Natur alles alleine heilen könne und ein therapeutisches Eingreifen in einen Krankheitsprozess daher nicht notwendig wäre. Dieser Zugang ermöglichte es Skoda jedoch, Krankheitsverläufe unbeeinflusst beobachten zu können und als exzellenter Diagnostiker in die Medizingeschichte einzugehen. Auf diese Weise erfolgte freilich auch eine Abkehr von bisherigen therapeutischen Maßnahmen, man propagierte (zumindest für die Patientinnen und Patienten des Allgemeinen Krankenhauses) eine Nicht-Behandlung, die von Zeitgenossen durchaus als unterlassene Hilfeleistung und Verstoß gegen die Grundsätze ärztlichen Handelns verstanden wurde. Bezüge zu diesen immerhin zeitgenössischen Kritiken finden sich bei Seligmann - verständlicherweise? - nicht, ebenso wenig in späteren Berichten der Wiener Medizingeschichtsschreibung über diese Epoche, vielmehr wurde genau dieses Herangehen als Grundlage für das Aufblühen der Wiener Medizinischen Schule des 19. Jahrhunderts gerühmt, auch in den Arbeiten von Erna Lesky.

Seligmanns Texte über die beiden „Wiener medizinischen Schulen“ lesen sich, auch unter Berücksichtigung der zeitgenössischen Praxis der Geschichtsschreibung, wie öffentlichkeitswirksam aufbereitete Jubelschriften: weitgehend unkritisch und das aktuell hohe wissenschaftliche Niveau unterstreichend. Dies evoziert die Vorstellung eines durch lange Jahre des Bemühens um eine akademische Anstellung im Allgemeinen und in der Medizingeschichte im Besonderen zermürbten, an sich jedoch kompetenten Historikers. Dabei stellt sich erneut die Frage nach dem Ausblenden gewisser Aspekte der zu diesem Zeitpunkt noch einigermaßen rezenten Vergangenheit. Es drängt sich die Annahme auf, dass damit die Strategie verfolgt wurde, die durch Stiffts Maßnahmen bewirkten Veränderungen als außergewöhnliche Leistungen der Medizinischen Fakultät darzustellen, aber weitgehend zu verschweigen, dass diese von einer Institution übernommen wurden, die im Vormärz offenbar ihrerseits als inkompatibel mit dem staatlichen Erwartungshorizont erschien.

Was hat all das aber mit der Frage nach dem medizinhistorischen „Schreiben über Josephinismus" im 20. Jahrhundert zu tun?

\section{MYTHOS GÉRARD VAN SWIETEN}

Gérard van Swieten ist eine zentrale Figur in den Arbeiten von Erna Lesky und ihren Vorgängern, einer der „großen Männer“ dieser Zeit, der nicht nur „Wien“ sondern auch „die Weltmedizin“ höchst nachhaltig prägte ${ }^{53}$ - der Begründer der „,ersten“ oder ,„älteren“ Wiener Schule der Medizin, der Reformator nicht nur des medizinischen Unterrichts; jener, der eine völlig neue Medizin etablierte, Wien einen Innovationsschub erleben ließ und diesen Ort

53 Diese Darstellungsweise zieht sich durch sämtliche Publikationen von Erna Lesky. Z. B. Erna LesKY, Vorwort, in: EAD. (Hg.), Wien und die Weltmedizin. Graz 1974, 9-10; EAD., Gerhard van Swieten, Auftrag und Erfüllung, wie Anm. 15, 30. 
ein für alle Mal zu einem Mekka der Medizin machte; ein Mann, dessen „Erscheinen“ eine solche „Strahlkraft“ und Nachhaltigkeit hatte, dass die Zeit davor, wie erwähnt, als dunkles Kapitel einer spekulativen Medizin wahrgenommen wurde, wodurch auch der Eindruck gefestigt wurde, dass eine Auseinandersetzung mit der Medizin in Wien vor diese „Zeitenwende“ völlig überflüssig wäre. Gérard van Swieten selbst wurde damit zum Epochenmacher. Diese Darstellungsweise erscheint als festgefügter und nahezu unausweichlicher Topos.

Gérard van Swieten ist in den Sitzungsberichten der Sanitätshofdeputation nie als Teilnehmer angeführt, wohingegen der frühere persönliche Arzt von Karl VI., Paul Michael von Zwenhoff, sehr wohl als medizinischer Experte diesem Gremium angehörte. Erna Lesky geht in Zusammenhang mit dieser Tatsache davon aus, dass van Swieten dennoch auf jeden Fall an der Arbeit dieser Kommission wesentlich beteiligt war - selbst wenn diese Funktion in der Literatur nicht erwähnt wird. ${ }^{54}$

Wenn wir die bisherige Literatur über diesen großen europäischen Arzt überblicken, so werden wir ihn charakterisiert finden als Leibarzt der Kaiserin, als Reformator der Wiener Universität und ihrer medizinischen Fakultät, als Zensor und Präses der Hofbibliothek, nirgends aber als den sanitären Organisator und obersten Sanitätschef eines großen Reiches. Es ist schmerzlich, festzustellen, daß dieses Versäumnis, die Würdigung seiner ärztlichen Hauptleistung, heute nach dem Verlust der zentralen Archivkörpers, der Hofkanzlei und des Staatsrates, nicht mehr nachzuholen ist.

Lesky argumentiert, dass sich in den Protokollbänden der Sanitätshofdeputation immer wieder die Bemerkung findet „Note an Baron van Swieten“: Daher geht sie davon aus,

... daß diese Art des Verkehrs den Schluss zulässt, daß van Swieten der Sanitäts- Hofdeputation als Mitglied nicht angehört hat, sondern sie von außen wie irgendeine andere Hofbehörde wie beispielsweise die Hofkammer beriet.

Dies scheint nachvollziehbar, aber ob sich daraus die von Lesky angenommene „Hauptleistung“ eines „obersten Sanitätschefs“ ableiten lässt, darf bezweifelt werden.

Eine der wesentlichsten Errungenschaften, die Gérard van Swieten zugeschrieben werden, ist die Reform des medizinischen Studiums in Wien sowie die Einführung des Unterrichts am Krankenbett. Die Reformen, die von ihm umgesetzt wurden, waren freilich bereits 1719 ausgearbeitet worden. Allerdings gerieten die Verhandlungen offenbar für längere Zeit ins Stocken, und lediglich die Statuten für die medizinische Fakultät wurden erneuert. ${ }^{55}$ Die Gründe hierfür waren bislang noch nicht Gegenstand einer ausführlichen Analyse, wiewohl

54 Lesky, Österreichisches Gesundheitswesen, wie Anm. 3, 41f.

55 Sonia Horn, „ein wohl auffgerichtes theatrum anatomicum “. Anatomischer Unterricht für nichtakademische Heilkundige an der Wiener medizinischen Fakultät im 18. Jahrhundert. In: Karin STUKENBROCK / Jürgen Helm (Hg.), Tagungsband des Internationalen Abraham Vatter Symposion Wittenberg 2001. Stuttgart 2003, 193-195. 
es van Swietens organisatorischen Fähigkeiten und einer günstigen politischen Konstellation zu verdanken sein dürfte, vielleicht aber auch lediglich „der Gunst der Stunde“, dass die geplanten Reformen schlussendlich umgesetzt wurden.

Die Aktenüberlieferung der Studienhofkommission im Allgemeinen Verwaltungsarchiv zeigt im Hinblick auf die Studienreformen, dass Gérard van Swieten ein engagierter und kompetenter „Universitätsmanager" war, der offenbar die Fähigkeit besaß, komplizierte Sachverhalte, die die Doktoren der Medizinischen Fakultät in wohlgesetztem Latein ausführlich beschrieben, konzise auf den Punkt zu bringen; in klarem Französisch wurden diese ohne Informationsverlust zusammengefasst und in Handbillets weitergegeben. Van Swieten wusste auch um besondere Wünsche einzelner Personen, vermochte wiederholt allseits attraktive Lösungen zustande zu bringen und dabei meist auch noch finanzschonend vorzugehen. Die für die Habsburgischen Länder 1770 in Kraft getretene Sanitäts- und Kontumazordnung war von großer Relevanz, beruhte jedoch auf einem Modell, das sich über mehrere Jahrhunderte hinweg im Kontext der Wiener und der Prager Medizinischen Fakultäten entwickelt hatte. Auch hier hatte die Medizinische Fakultät Gérard van Swieten auf die enormen Belastungen aufmerksam gemacht, die die Verwaltung des Gesundheitswesens für die Fakultät bedeutete, was auch in den ersten Planungsentwürfen für die Umsetzung der anvisierten Reformen thematisiert wurde. ${ }^{56}$

Diese Entwürfe enthalten sehr viele elaborierte und klar strukturierte Vorschläge, die sich in erster Linie auf die Ausbildung und die Befugnisse verschiedener Gruppen von professionellen Heilkundigen beziehen, die sowohl im zivilen als auch im militärischen Bereich tätig waren. Die Ausgliederung der Verwaltung des Gesundheitswesens aus den Agenden der Medizinischen Fakultäten (zumindest) von Prag und Wien ist hierbei ein gewichtiger Aspekt. Es ist demnach davon auszugehen, dass van Swieten dies auch gegenüber der Sanitätshofdeputation vertreten und in diesem Zusammenhang auch an deren Entscheidungen mitgewirkt hat. Zu diskutieren wäre allerdings, ob dies als seine „Hauptleistung“ zu werten ist, vor allem im Hinblick auf die Umsetzung der Reformen im universitären Bereich.

Die besonderen Leistungen von Gérard van Swieten liegen in einem Bereich, der im englischsprachigen Raum mit herding cats umschrieben wird, dem des „Universitätsmanagements". Die von ihm umgesetzten Konzepte waren bereits weitgehend ausgearbeitet, seine Leistung lag in der konzisen Formulierung der Probleme und der Koordinierung der umsetzenden Akteure. Zudem brauchte es auch die Fähigkeit überzeugender Vermittlung, was sich aus den zahlreichen erhaltenen Stellungnahmen und Lösungsvorschlägen, die Gérard van Swieten verschiedenen Interessensvertretern unterbreitete, nachvollziehen lässt. Auch eine entsprechende Kommunikationsfähigkeit sowie das Erkennen, Abwägen und Einsetzen von Einzelinteressen zeigt sich in diesen Dokumenten.

Gérard van Swieten ist sicher in vielfacher Hinsicht eine wesentliche Figur. Warum aber wurde er zu einer Heldengestalt gemacht, warum wurden ihm Leistungen zugeschrieben, die man bei solider Recherche in den Quellen, aber durchaus auch in der vorhandenen Sekundärliteratur historiographisch wesentlich seriöser hätte aufbereiten können? Und warum hat

56 Horn, Entstehung der medizinisch-chirurgischen Akademie, wie Anm. 11, $219 f$. 
Gérard van Swieten schließlich als einer der „Großen aus Wien“ auch in den Darstellungen von Eduard Winter eine ähnliche Funktion als „Epochengrenze“?

\section{VI „SPEKULATIVE MEDIZIN“ UND „UNTERRICHT AM KRANKENBETT“}

Die Medizin selbst kann für die Zeit vor van Swieten - und auch frühere Epochen - nicht als „(rein) spekulativ“ bezeichnet werden, wie dies oft geschehen ist. Grundlage des medizinischen Denkens war bis in die Mitte des 19. Jahrhunderts das hippokratisch-galenische Konzept. Die genannten Proponenten der vormärzlichen Medizin sind daher durchaus in diesem Lehrgebäude beheimatet. Mit der Krasenlehre finden sich hippokratisch-galenische Elemente auch in den Theorien von Carl von Rokitansky.

Angesichts dieser Konstellation ist es nicht nachvollziehbar, warum Erna Lesky diese Kategorie mit einem einigermaßen negativen Tenor Andreas von Stifft als Vertreter einer „Doktrin“ zuweist. Gérard van Swieten und seine Kollegen, aber auch sein Lehrer Hermann Boerhaave (1668-1738), vertraten im Grunde konservative, hippokratische Lehrmeinung und zeigten sich, etwa am Beispiel der weitgehenden Ablehnung der Pockenimpfung, als einigermaßen ,innovationsfeindlich“ - was für Stifft wiederum nicht zutrifft, da er diese befürwortete. Auffällig ist, dass Stifft auch bei Eduard Winter ähnlich charakterisiert und als Gegenmodell zu van Swieten aufgebaut wird.

Um aber eine so genannte „spekulative“ Herangehensweise an ein Krankheitsgeschehen definieren zu können, braucht es einen Bezugsrahmen, von dem aus Handlungen oder Konzepte als „spekulativ“ wahrgenommen und bewertet werden können. Insofern müssen medizinische Therapien, aber auch Beobachtungen der Natur und vieles andere, das mit Wissen im Sinn von Wahrnehmung, Interpretation und Anwendungsmöglichkeiten des Wahrgenommenen zu tun hat, aus dem zum jeweiligen Zeitpunkt relevanten Konzept und Wissensstand heraus verstanden werden, alles andere ist anachronistische Projektion. Somit muss man versuchen, einen Zugang dazu zu finden, ob etwa eine Beobachtung oder eine Therapie in ihrer Zeit selbst als ,innovativ“ verstanden wurde. ${ }^{57}$ In Bezug auf die Medizin ergibt sich hier klar, dass die hippokratisch-galenische Denkweise, die im 18. Jahrhindert relevant war, kaum als „spekulativ“ zu bezeichnen ist.

Auch der Unterricht am Krankenbett ist keine Einführung von Gérard van Swieten, denn ein solcher war in der ärztlichen Ausbildung an der Medizinischen Fakultät der Universität Wien von Beginn der Aufzeichnungen an vorgesehen..$^{58}$ Studenten mussten ihre

57 Vgl. dazu Hasok Chang, Is Water H2O? Evidence, Pluralism and Realism. Dordrecht 2012.

58 Sonia Horn, ,... damit sy in ain rechte erfahrenheit der practighen khummen. " Der praktische Unterricht für akademische Ärzte vor den Reformen durch Van Swieten. In: Helmuth Grössing / Sonia Horn / Thomas Aigner (Hg.), Wiener Gespräche zur Sozialgeschichte der Medizin, Vorträge des Internationalen Symposions an der Universität Wien 9.-11. November 1994. Wien 1996, 75-96; Michael Stollberg, Bedside Teaching and the Acquisition of Practical Skills in Mid-Sixteenth-Century Padua. In: Journal of the History of Medicine and Allied Sciences 69/4 (2014), 633-661. 
Promotoren zumindest ein Jahr lang bei der Betreuung von Patientinnen und Patienten begleiten. In Wien bestand das Problem eher darin, dass Studenten die eigenständige Betreuung von Kranken unerlaubt und im Hinblick auf ihren Ausbildungsstand zu früh durchführten. Auch die Betreuung von Patientinnen und Patienten in Krankenhäusern war üblich, etwa auch im Spital der Barmherzigen Brüder, für das eine Beschreibung desselben von 1626 vorliegt. ${ }^{59}$ Eine mehrjährige praktische Ausbildung erfolgte auch am 1737 gegründeten Dreifaltigkeitsspital, was jedoch nur den Originalquellen zu entnehmen ist, da keine Sekundärliteratur zu diesem Spital existiert. Dies ist insofern einigermaßen merkwürdig, als es gerade hier klare Vorgaben für die praktische Ausbildung für Studenten der Medizin und der Chirurgie gab. ${ }^{60}$ Auch hier ergibt sich die Frage nach dem Grund dieses Forschungsdefizits, immerhin geht es denn doch um einen nicht unbedeutenden Aspekt der „Wiener Medizin“.

Noch aufschlussreicher wird diese Frage, wenn man den Versuch unternimmt, die von Gérard van Swieten ins Leben gerufene „Medizinisch-Praktische Lehrschule“ am Bürgerspital unter die Lupe zu nehmen, also diese aus Originalquellen zu rekonstruieren, etwa anhand der Frage, wie die Kosten für die Einrichtungsgegenstände (Betten, Bettwäsche, Heilbehelfe ...) beglichen wurden. Noch schwieriger ist es herauszufinden, was die immerhin dreijährige praktische Ausbildung im Dreifaltigkeitsspital (die offenbar unter Anleitung von erfahrenen Ärzten, die auch der Medizinischen Fakultät angehörten, erfolgte) vom „Unterricht am Krankenbett“ in der von Gérard van Swieten (angeblich?) eingerichteten Medizinisch-Praktischen Lehrschule unterschied, vor allem in Bezug auf die Qualität dieser Ausbildung. Dieser Frage ist bereits Christian Probst 1972 nachgegangen, ohne zu einem klaren Ergebnis zu kommen: Es erscheint nicht nachvollziehbar, in welcher Form diese Schule am Bürgerspital existierte und warum schlussendlich Anton de Haen nach Wien berufen wurde, um diese Medizinisch-Praktische Lehrschule zu leiten. ${ }^{61}$

Daniela Wagner konnte jüngst auf der Basis von Archivmaterial rekonstruieren, was Gérard van Swieten unter diesem Unterricht konkret verstand. Demnach sollten Krankheiten, die im theoretischen Unterricht besprochen wurden, parallel dazu auch an Patientinnen und Patienten beobachtet werden. ${ }^{62}$ Dies unterscheidet sich denn doch von einem Unterricht „am Krankenbett“, für den die Patientinnen und Patienten nicht eigens ausgewählt wurden. Am Wiener Bürgerspital fanden seit 1721 so genannte „Collegia publica“ statt, bei denen den Studenten der Medizin und der Chirurgie, aber auch den Hebammenschülerinnen, Patientinnen und Patienten vorgestellt wurden. Während dieser „Collegia“ wurden die Krankheiten und die Therapien ausführlich erläutert. ${ }^{63}$ Der Unterschied zwischen dieser Art der medizi-

59 Sonia Horn, Der praktische Unterricht für akademische Ärzte, wie Anm. 58

60 Anordnung und Verfassung des Krankenspitals zur allerheiligsten Dreyfaltigkeit. In: Codex Austriacus, Suppl. Teil 5, 1740-1758. Wien 1777, 59-94.

61 Christian Probst, Der Weg des ärztlichen Erkennens am Krankenbett, 1: 1707-1787. Wiesbaden 1972, $106 f$.

62 Wagner, Gérard van Swieten und die Gründung der Kliniken, wie Anm. 14, 22-24.

63 Horn, Anatomischer Unterricht für nichtakademische Heilkundige, wie Anm. 55, 205. 
nischen Lehre und dem von van Swieten intendierten Unterricht an der Medizinisch-Praktischen Schule erscheint jedoch nicht substantiell.

1754 wurde das Dreifaltigkeitsspital mit dem Spanischen Spital, das sich im Gebäude des heutigen Erzbischöflichen Priesterseminars Wien befindet, zum „Unierten Spital“ vereinigt und die Medizinisch-Praktische Lehrschule aus dem Bürgerspital dorthin verlegt. Auffällig ist die starke Ähnlichkeit der Beschreibung des Unterrichtes am Krankenbett, wie Anton de Haen ihn 1754 abgehalten haben soll, mit jenem, den Ferdinand Illmer von Wartenberg (gest. 1698) am Spital der Barmherzigen Brüder um 1695 erteilte.

Niemallen kame er zu uns in das Spital, wo ihme nicht viel vorwarteten, oder aber nachfolgeten auß seinen Discipuln, denen er wehrender Ordination bald diese oder jene sinnreiche Frag von deren Kranckenzuständ auffgabe, erforschte ihre Muthmassung als ein sorgfältiger Lehrmeister; und so dieselben nach ihren Gedancken ihre Meinung und Antwort entdecketen, als dann gab er seine weiseste Resolutiones und Aussprüch. ${ }^{64}$

Schon Früh um 6 Uhr ist Haen, des Winters sowohl als des Sommers, im Krankenhause, und um 8 Uhr versammeln sich seine Zuhörer, diese führt er sogleich zum Krankenbette, erklärt mit der grössten Genauigkeit den vorliegenden Fall, erforscht die Ursachen mit dem ihm eigenen Scharfsinn, entwickelt sorgfältig die Symptome. Nun untersucht der Zuhörer den Kranken selbst, was er bemerkt hat sagt er dem Lehrer ins Ohr, dieser sammelt alle Stimmen und zeigt hernach öffentlich an, sowohl was falsch als auch was recht bemerkt worden ist. ${ }^{65}$

Klar wird hierbei jedoch auch, dass diese Beschreibung nicht unbedingt dem entspricht, was Gérard van Swieten beabsichtigt hatte, denn in beiden Fällen handelt es sich eher nicht um ausgewählte Krankheitsfälle.

Der bedeutende Wiener Medizinhistoriker Theodor Puschmann ${ }^{66}$ thematisiert in seiner Geschichte des medizinischen Unterrichts auch den „praktischen Unterricht“ bzw. den „Unterricht am Krankenbett“. Er erklärt, dass dieser an sich schon lange existierte und auch im Kontext der Universitäten von Anfang an betrieben wurde. Seiner Meinung nach sind jene im Irrtum, die behaupten, es hätte keinen praktischen Unterricht in der akademischen Ausbildung von Ärzten gegeben. Seine Interpretation geht dahin, dass dieser praktische Unterricht eben nicht unmittelbar an den Universitäten erfolgte, sondern „außerhalb“ organisiert

64 Horn, Praktischer Unterricht für akademische Ärzte, wie Anm. 58, 93.

65 Gustav LöвEL, Geschichtliche Notizen über das medizinische Clinicum der Wiener Universität, 2. In: Wiener Medizinische Wochenschrift, 21 (1871) [Nr. 27], 662.

66 Theodor Puschmann (1844-1899) studierte Medizin in Berlin, Marburg, Wien und München; 1878 Habilitation im Fach Geschichte der Medizin an der Universität Leipzig, 1879 außerordentlicher Professor für Medizingeschichte in Wien, 1888, 1898/99 Dekan der medizinischen Fakultät. Puschmann hinterließ einen Teil seines Vermögens der Universität Wien zur Einrichtung einer Bibliothek und eines Museums für Medizingeschichte. Den Großteil vermachte er jedoch der Universität Leipzig zur Gründung eines medizinhistorischen Instituts, das als das erste seiner Art gilt. 
wurde, und dass die Studenten die Fakultätsmitglieder bei den Krankenbesuchen sowohl im Spital als auch anderswo begleiten mussten. Diese Argumentation erscheint allerdings wenig überzeugend, gerade wenn man die begleitenden institutionellen Bezüge berücksichtigt, denn dieser „Unterricht am Krankenbett“ war durchaus in konkrete institutionelle Rahmen eingebettet, wie dies die Beispiele von Padua und Wien zeigen. ${ }^{67}$

Zwischen den medizinischen Fakultäten und den Krankenhäusern, in denen dieser praktische Unterricht durchgeführt wurde, bestanden personelle und institutionelle Verbindungen. Besonders deutlich wird dies am Dreifaltigkeitsspital, in dem auf der Basis von Stiftungen, die durch Universitätsangehörige getätigt worden waren, arme und vor allem akut Erkrankte sowohl internistisch als auch chirurgisch betreut wurden. Die dreijährige Ausbildung von Studenten der Medizin und der Chirurgie als „Praktikanten“ war klar geregelt und umfasste auch die Durchführung von Obduktionen. Den „Praktikanten“ standen auch ein $\mathrm{Mu}$ seum und eine Bibliothek zur Verfügung, ein tägliches, zweistündiges Studium in diesen Räumen war im Tagesablauf vorgesehen. Die Leitung der verschiedenen Abteilungen und der Unterricht wurden von Professoren und Doktoren der Medizinischen Fakultät wahrgenommen. Verglichen mit den Berichten über die „Medizinisch-Praktische Lehrschule“ ergibt sich daraus keine besonders auffälliger Unterschied im Hinblick auf die medizinische Ausbildung - außer dass die Praktikanten des Dreifaltigkeitsspitales wahrscheinlich wesentlich mehr Patientinnen und Patienten zu Gesicht bekamen und auch wesentlich stärker in die Krankenbetreuung eingebunden waren, als die Studenten an der Medizinisch-Praktischen Lehrschule. 1756 hielt De Haen in seiner Antrittsrede als Professor der medizinischen Klinik fest, dass die Studenten der Medizin mit seinem Unterricht bislang äußerst zufrieden gewesen seien und ihn sogar gebeten hätten, auch während der Sommermonate den praktischen Unterricht weiter zu führen. Mit Verweis darauf, dass die Studierenden diese Ferien zur Erholung benötigen würden, habe er diese Anfrage jedoch abgelehnt. ${ }^{68}$ Dies könnte aber auch als Wunsch der Studierenden nach einer intensiveren praktischen Ausbildung interpretiert werden, eventuell auch in Zusammenhang mit den Möglichkeiten, die den Praktikanten zuvor am Dreifaltigkeitsspital offen gestanden waren. Hinzu kommt, dass Gérard van Swieten die Meinung vertrat, dass die Studenten zu früh und ohne ausreichendes theoretisches Wissen in die praktische Ausbildung gingen. In diesem Zusammenhang wird auch der Vergleich mit einer handwerklichen Ausbildung bemüht. ${ }^{69}$

Auf diese Weise würde man im Gegensatz zu den üblichen Narrativen sogar zu dem Eindruck kommen, dass die Quantität, aber vielleicht auch die Qualität, der praktischen Ausbildung in der Medizin reduziert wurde. Dies würde allerdings der Situation in der Ausbildung von Hebammen in Wien nach 1748 entsprechen. Auch hier wurde der Umfang des vermittelten Wissens reduziert und das Tätigkeitsspektrum sowie die Handlungsspielräume von

67 Theodor Puschmann, Geschichte des medicinischen Unterrichts von den ältesten Zeiten bis zur Gegenwart. Leipzig 1889, $341 \mathrm{f}$.

68 LöвEL, Geschichtliche Notizen 2, wie Anm. 65, 689.

69 WaGNeR, Gérard van Swieten und die Gründung der Kliniken, wie Anm. 14, 23. 
Hebammen eingeschränkt. ${ }^{70}$ Von Gérard van Swieten als dem Begründer des praktischen Unterrichts in Wien ist bei Puschmann nichts zu lesen. Die Darstellungen Puschmanns - eines nicht nur in seiner Zeit anerkannten, „institutionalisierten“ und handwerklich versierten Wiener Medizinhistorikers - wurden von seinen Nachfolgerinnen und Nachfolgern inhaltlich offenbar nur sehr unzulänglich rezipiert. Stattdessen wurden mit historischen Quellen relativ leicht widerlegbare Falschaussagen getätigt, was denn doch Verwunderung hervorruft und nach den historiographiegeschichtlichen Ursachen solch einer Konstellation fragen lässt.

Mit größter Vehemenz in Frage gestellt wird die Gérard van Swieten zugeschriebene medizinische Innovation, wenn bei Christian Propst nachgezeichnet wird, dass wenige Jahre nach dem Tod von van Swieten Reformen des Studiums und insbesondere des praktischen Unterrichts als dringend notwendig erachtet wurden. Propst beschreibt die „Methodik“, die Gérard van Swieten und Anton de Haen vertraten, als zu ihrer Zeit bereits „erstarrt“ ${ }^{71}$ Im Hinblick darauf verliert auch die Aussage von Albrecht von Haller ihre Härte, der meinte, dass sein Studienkollege eher ein unterdurchschnittlicher Mediziner wäre und alles andere als innovativ. ${ }^{72}$ An „innovativem“ medizinischem Wissen kann die „Größe“ von Gérard van Swieten aus zeitgenössischer Sicht also eher nicht festgemacht werden.

Diese dominierenden Narrative wurden jedoch von den nachfolgenden Medizinhistorikern und Erna Lesky weitgehend übernommen. Freilich hat Theodor Puschmann durchaus versucht, auch andere Epochen zu berücksichtigen. Es stellt sich hierbei die Frage, ob dies Schwierigkeiten mit Vertretern der Medizinischen Fakultät verursachte, die jedoch in den Ausführungen nicht eigens angeführt, aber mit der möglicherweise verspäteten Verleihung einer ordentlichen Professur in Zusammenhang gebracht werden können. ${ }^{73}$

Der Topos der ersten und zweiten Wiener medizinischen Schule und der eingeschränkte Blick auf die Medizinisch-Chirurgische Akademie, einer immerhin zentralen Institution im „josephinischen“ Gesundheits- und Bildungswesen, die Datumsgrenze „van Swieten“ und die Definition medizinischer Konzepte in der Zeit „davor“ als „spekulativ“ - all diese Erzählelemente wurden auch von Max Neuburger weitergetragen. ${ }^{74}$ Wesentlich bekannter sind

70 Sonia Horn, Wiener Hebammen 1643-1753. In: Studien zur Wiener Geschichte 59 [= Jahrbuch des Vereins für Geschichte der Stadt Wien] (2003), 82-86.

71 Рвовsт, Der Weg des ärztlichen Erkennens, wie Anm. 61, 213-217.

72 Rina Knoeff, Herman Boerhaave at Leiden: Communis Europae Praeceptor. In: Ole Peter Grell (Hg.), Centres of Medical Excellence? Medical Travel and Education in Europe, 1500-1789. Farnham 2010, 269-289, hier 279f.

73 Gabriela Sснміdт, Theodor Puschmann und seine Verdienste um die Errichtung des Faches Medizingeschichte an der Wiener Medizinischen Fakultät. In: Andreas Frewer / Volker Roelke (Hg.), Die Institutionalisierung der Medizinhistoriographie. Entwicklungslinien vom 19. ins 20. Jahrhundert. Stuttgart 2001, 91-102.

74 Max Neuburger (1868-1955) studierte Medizin in Wien; Promotion 1893; Schüler von Theodor Puschmann. Habilitation in Medizingeschichte 1898, 1904 außerordentlicher Professor, 1917 ordentlicher Professor für Medizingeschichte. 1914 Gründung des Instituts für Geschichte der Medizin an der Universität Wien, Übersiedlung in das Gebäude des Josephinums und Aufbau der Sammlungen. 1939 Emigration nach London, wo er am Wellcome Institute for the History of Medicine seine Forschungsar- 
die Publikationen von Erna Lesky über die „erste und zweite Wiener Schule“ und eben über Gérard van Swieten. ${ }^{75}$ Diese waren offenbar so prägend, dass vielfach bis heute die Meinung besteht, dieses Thema sei bereits erschöpfend behandelt.

Daniela Wagner hat in ihrer kürzlich abgeschlossenen Masterarbeit am Institut für Österreichische Geschichtsforschung die komplexen verwaltungsgeschichtlichen Aspekte der Gründung der Medizinischen-Praktischen Lehrschule ausführlich dargestellt und dies in den Kontext der "Verwaltungsverdichtung " dieser Epoche gesetzt. ${ }^{76}$ Klar herausgearbeitet wurde hierbei auch, dass es um ein Eingreifen des Staates in den universitären Bereich ging und um die Aneignung von Stiftungsgeldern, die für die Umsetzung von staatlichen Zielen eingesetzt wurden. Dies gilt auch für das Dreifaltigkeitsspital, dessen Finanzierung auf Stiftungsgeldern beruhte. Diese Institution wurde 1754 mit dem Spanischen Spital zum „Unierten Spital“ vereinigt, woraus eine Institution wurde, die als staatliche Einrichtung anzusehen ist. Mit der Verlegung der "Medizinisch-Praktischen Lehrschule“ an dieses Spital wurden sowohl finanzielle als auch verwaltungstechnische Probleme bereinigt. Dies bedeutete jedoch auch, dass der medizinische Unterricht aus einer Institution, die auf einer Stiftung von Universitätsangehörigen beruhte und mit der medizinischen Fakultät in enger Verbindung stand, in eine „staatliche“ Einrichtung verlegt wurde, indem die Stiftung selbst in diese Einrichtung übergeleitet wurde. Dies würde sich durchaus logisch in die „Verstaatlichung" des Universitäts- und Bildungswesens einfügen. Im Grunde wäre damit auch die allgemeine (begriffliche) Verwirrung um den praktischen Unterricht, den klinischen Unterricht, den „Unterricht am Krankenbett“, oder wie auch immer die Terminologie lauten mag, für Wien gelöst. Wahrscheinlich ging es nicht um das, was hier "getan“ wurde, sondern darum, wie diese Vollzüge verwaltet wurden und in welchen Zuständigkeitsbereich dies fiel - man könnte das Thema also in die bekannte Transformierung der habsburgischen Universitäten zu staatlichen Ausbildungsinstitutionen einreihen.

Angesichts von, plakativ ausgedrückt, Barrikaden stürmenden Horden von (Medizin-) Studenten, die die Freiheit von Forschung und Lehre forderten und gegen die staatliche Einflussnahme auf den Wissenschaftsbetrieb ankämpften, ist es geradezu verständlich, dass die „Fortschritte“ eines verstaatlichten Universitäts- und Bildungswesens ab 1848 historiographisch ganz besonders herausgearbeitet werden sollten bzw. wurden. Die praktische Ausbildung ist für die medizinische Tätigkeit immerhin ein sehr wesentlicher Aspekt und bietet sich daher in besonderem Maß als Thema an, um klar zu machen, dass die damit verbundene Qualität der Ausbildung und das sich hierauf gründende hohe Niveau des Wissens erst möglich wurden, als der Staat und sein Bildungsmanager Gérard van Swieten diese Institutionen

beiten fortsetzen konnte. 1948 nahm er seine Tätigkeit an der University of Buffalo auf, 1952 Rückkehr nach Wien.

75 Lesky / WANDruszKa (Hg.), Gerhard van Swieten und seine Zeit, wie Anm. 15; sowie Lesky (Hg.), Wien und die Weltmedizin, wie Anm. 51.

76 Vgl. dazu Michael Hocheduinger, Verfassungs-, Verwaltungs- und Behördengeschichte der Frühen Neuzeit. Vorbemerkungen zur Begriffs- und Aufgabenbestimmung. In: ID. / Thomas Winkelbauer (Hg.), Herrschaftsverdichtung, Staatsbildung, Bürokratisierung. Verfassungs-, Verwaltungs- und Behördengeschichte der Frühen Neuzeit. Wien 2010, 21-85. 
übernahmen und transformierten. Um dies deutlicher zu machen, musste auch die „Zeit davor" als dunkles Kapitel einer spekulativen Medizin konstruiert und über Themen wie die praktische Ausbildung am Dreifaltigkeitsspital geschwiegen werden. Insofern ist auch nachvollziehbar, dass es zu dieser Institution und vielen anderen medizin-, bildungs- und sozialpolitischen Themen keine oder kaum Sekundärliteratur gibt bzw. erst in den letzten Jahrzehnten genauer auf einschlägige Narrative und Geschichtskonstruktionen geachtet wird. Andererseits ist dies aber auch nicht nachvollziehbar, denn durch solide historische Arbeit an Quellen hätte dies schon vor geraumer Zeit hinterfragt werden können, sofern man nicht bewusst oder unbewusst - an Narrativen festhält, die als Reaktion auf gesellschaftspolitische Entwicklungen entstanden sind und die vorderhand für die eigene Meinung zu aktuellen Themen der Gesellschaftsentwicklung und Universitätspolitik Relevanz haben.

Die (Medizin-)Geschichtsschreibung des 19. Jahrhunderts ist demnach nicht nur als reine „Fortschrittslegitimation“ zu verstehen, sondern sehr wohl auch als bildungs- und universitätspolitische Botschaft an mehr oder weniger aufsässige Mediziner. Die Errungenschaften staatlicher Universitätsmedizin kommen so in krassen Gegensatz zu den Unzulänglichkeiten der vorangegangenen Epochen, und die Konstruktionen eines Helden und einer Schwelle bietet sich an.

\section{VII „NATIONALISMUS“}

Erna Lesky widmete sich freilich auch Aspekten, die in den Bereich der Forschungen zu „Osteuropa“ fallen, welche bei Fritz Valjavec und Eduard Winter eine wichtige Rolle spielten. Der Widerhall von deren Arbeiten in der tschechoslowakischen, ungarischen und jugoslawischen Historiographie wird in anderen Beiträgen des vorliegenden Buches dargestellt.

1972 fungierte Lesky neben Strahinja K. Kostić, Josef Matl und Georg Rauch als Herausgeberin eines Tagungsbandes mit dem Titel Die Aufklärung in Ost- und Südosteuropa ${ }^{77}$ steuerte jedoch selbst keinen Aufsatz bei. Da das sehr kurz gehaltene Vorwort nicht unterschrieben ist, bleibt Leskys Beitrag nicht nachvollziehbar. Zwei Jahre zuvor hatte sie eine etwa 70 Seiten starke Monographie über Johann Evangelist Purkyně veröffentlicht, in der in erster Linie der Zusammenhang von Purkyněs universitärer Tätigkeit mit seinem Engagement im tschechischen Nationalismus thematisiert wird. ${ }^{78}$ Hier entwirft sie das Bild eines von Nationalismus geprägten Physiologen, der in Breslau tätig war und dem durch den ebenfalls nationalistisch geprägten und unter dem Einfluss von Bernard Bolzano stehenden Minister Leo Thun-Hohenstein die Rückkehr in sein Heimatland und an die Universität Prag ermöglicht wurde. Beide Wertungen stehen im Gegensatz zu den Studien Eduard Winters, in denen Purkyně und Thun als Paradebeispiele für den böhmischen Landespatriotismus gelten, der die Va-

77 Erna Lesky / Strahinja Kostič / Josef Matr / Georg Rauch (Hg.) Die Aufklärung in Ost- und Südosteuropa. Aufsätze, Vorträge, Dokumentationen. Köln - Wien 1972.

78 Erna Lesky, Purkyněs Weg. Wissenschaft, Bildung und Nation. Wien 1970. 
terlandsliebe mit einer mehrsprachigen, eben nicht ethnonational definierten Identität verband. ${ }^{79}$ Purkyně wird bei Lesky als alternder Professor dargestellt, dessen Leistungen nicht mehr dem aktuellen Stand entsprachen, wie dies von Carl Rokitansky festgestellt worden war. Dennoch sei man „von Wien aus“ mit Purkyně sehr rücksichtsvoll, geduldig und elegant umgegangen. Die These des Purkyně-Biographen Henry J. John, ${ }^{80}$ dass man den national gesinnten Purkyně von Wien aus stark eingeschränkt hätte, weist sie mit zahlreichen Quellenbezügen zurück, die im Anhang des Buches auch in einer Transkription wiedergegeben sind. ${ }^{81}$

Eine aufschlussreiche Quelle ist in diesem Zusammenhang ein äußerst kritisches Feuilleton mit dem Titel Nationale Wissenschaft, das in der Wiener medizinischen Wochenschrift vom 3. Mai 1865 veröffentlicht worden war. ${ }^{82}$ Hier wird die Tatsache, dass Purkyněs Schüler, der offenbar hoch qualifizierte Physiologe Johann Czermak, nicht als Nachfolger seines Lehrers im Land gehalten werden konnte, sondern einem Ruf nach Jena folgte, mit „czechischem Marasmus“ begründet - einer Kombination aus Nationalismus, damit verbundenem kulturellem Verfall und Beharrungstendenzen eines greisen Ordinarius. Dies ist auch mit dem Hinweis verbunden, dass Czermak fähig gewesen wäre, sowohl in Tschechisch als auch in Deutsch zu unterrichten und er insofern durchaus den sprachlichen Anforderungen einer solchen Stelle gewachsen gewesen wäre. ${ }^{83}$ Lesky berichtet ebenfalls über diese Thematik und arbeitet auf der Basis eines Briefwechsels von Purkyně und Czermak die sehr persönlichen Aspekte dieses Konfliktes eines Schülers mit seinem Lehrer heraus, der nichts davon wissen will, zurückzutreten und ihn als Nachfolger zu akzeptieren.

Auch neuere Studien sehen dieses Thema eher als persönliches Problem eines greisen Wissenschafters mit seinem jüngeren Kollegen und potentiellen Nachfolger, weniger als eines, in dem Nationalismus eine zentrale Rolle spielen würde. ${ }^{84}$ Vor dem Hintergrund dieses Beispiels wird ansatzweise deutlich, dass gerade die historiographische Generation von Winter und Valjavec einen grundsätzlich „nationalistischen“ Blick auf den Josephinismus - und andere Epochen - (in welche Richtung dieser auch gehen mag) gefördert und

79 Eduard Winter, Johann Evangelist Purkinje 1787-1869. In: Eduard Winter / Günther JAROsch, Wegbereiter der deutsch-slawischen Wechselseitigkeit. Berlin 1983, 235-244. Allgemein kritisch-fundiert zur Verklärungstendenz, die der Rede vom „Landespatriotismus“v. a. bei deutschböhmischen und österreichischen Historikern mitunter innewohnte: Jiř́ RAK, Welche Sprache sprechen die Bohemisten? In: Brücken Neue Folge 8 (2000), 59-69.

80 Henry John, Jan Evangelista Purkyně, Czech Scientist and Patriot 1787-1860. Philadelphia 1959.

81 Lesky, Purkyněs Weg, wie Anm. 78, 29-49.

82 P. B. [anonym], Nationale Wissenschaft. In: Wiener medizinische Wochenschrift, Jg. 15, Nr. 35 (1865), 613-617. Ich danke Prof. Ludmila Hlaváčková und Karel Černý vom medizinhistorischen Institut der Universität Prag für diesen Hinweis.

83 Vlg. dazu die ausführliche Darstellung des politisch-nationalen Rahmens bei Tatjana BukLIjAs, The Politics of Fin-de-Siècle Anatomy. In: Jan Surmann / Mitchell Ash (Hg.), The Nationalization of Scientific Knowledge in the Habsburg Empire, 1848-1918. Basingstoke 2012, 209-244.

84 Vladislav Kruta, Fysiologický ústav J. N. Czermaka v Praze. K historii fysiologie v Praze 1860-1865 [Das physiologische Institut in von J. N. Czermak in Prag. Beiträge zur Geschichte der Physiologie in Prag 1860-1865]. In: Československá fysiologie 22/2 (1973), 93-114. Auch hier danke ich Ludmila Hlaváčková und Karel Černý für den Hinweis und die inhaltliche Erläuterung. 
konsolidiert hatten, was jedoch in neueren Studien als wenig bis nicht relevant erscheint bzw. widerlegt wurde.

\section{PR, POLITIK UND „DAMNATIO MEMORIAE“}

Die bisher behandelten Topoi wurden im Laufe der - hinreichend langen - Wiener Medizingeschichtsschreibung konstruiert, festgeschrieben und weitergegeben. Heute taugen sie in vortrefflicher Weise den Zwecken der Öffentlichkeitsarbeit, und es ist nicht auszuschließen, dass dies von Beginn an der Auftrag der Wiener Medizinhistoriographie war.

Erna Lesky bezieht sich in ihren Arbeiten häufig auf Eduard Winter und Fritz Valjavec. Es ist anzunehmen, dass sie deren Vorstellungen von „Josephinismus“ übernommen und ihre Darstellungen darin eingebettet hat. Ähnlichkeiten zeigen sich bei der Betonung einer Art von „Epochengrenze“, die durch das Erscheinen des „großen Mannes“ Gérard van Swieten beinahe exakt zur Mitte des 18. Jahrhunderts definiert wurde und kaum Blicke in die Zeit „davor“ zuließ, nämlich in die Zeit der in den Publikationen häufig als „spekulative“ Heilkunde beschriebenen Medizin. Das weitgehende Schweigen über das Josephinum erinnert an die begrenzten Darstellungen bei Winter und Valjavec.

Der "Josephinismus“ im Gesundheits- und Sozialwesen ist in einigen Bereichen immer noch eine terra incognita. Seitens der Medizingeschichte stellt sich die Frage, warum einzelne Themenbereiche aus der Darstellung ausgeklammert wurden, wie etwa die wissenschaftliche und gesellschaftliche Bedeutung des Josephinums. Indizien deuten auf die vormärzliche damnatio memoriae einer politisch nicht unbedeutenden Institution hin, Ähnliches könnte für die Fixierung auf das „humanitäre“ Motiv des Kaisers bei der Gründung des Allgemeinen Krankenhauses gelten. Immerhin entzieht sich eine solch monumentale Einrichtung dem Vergessen, sie bietet sich dabei aber zugleich auch für einen angepassten Interpretationsrahmen an: Das Allgemeine Krankenhaus wurde schlussendlich in der institutionellen Memoria zu jener Institution, in der die „Wiener Schulen“ verortet waren und ihre Erfolge feierten.

Eduard Winter hat in seinen Publikationen über den Josephinismus das Gesundheits- und Sozialwesen im Grunde nicht thematisiert. Umso interessanter erscheint seine theologische Habilitationsschrift Die Gesundheitsfürsorge auf dem Lande als Ausgangs- und Mittelpunkt der ländlichen Wohlfahrtspflege in ihrer pastoralen und sozialen Bedeutung (Prag 1922). In dieser schlägt er als „pastorale Lösung“ der aktuellen, sehr ausführlich dargestellten und präzise analysierten Probleme im Gesundheits- und Sozialwesen der jungen Tschechoslowakei ein System vor, das starke Ähnlichkeit mit jenem aufweist, das im Josephinismus etabliert wurde und noch lange wirksam geblieben war. Die staatlich ausgebildeten und finanzierten Pfarrer hatten hierbei eine wesentliche Funktion, indem sie das System vor Ort organisierten und administrierten. Von ,josephinischen“ Pfarrern wurde erwartet, dass sie sich mit ihrer ganzen Person in den Dienst der Gemeinschaft stellten, hierfür wurde im Rahmen ihrer Ausbildung auch medizinisches Basiswissen vermittelt. Winter beschreibt diese Fähigkeit, sich aufopfern zu können, als persönliche Qualität, über die typischerweise besonders Frauen verfügen. 
Durch einen gezielten Religionsunterricht sollte das Ideal der tätigen Nächstenliebe bis hin zum Ignorieren persönlicher Bedürfnisse an Mädchen und junge Frauen vermittelt werden. Die diesbezügliche Leidensfähigkeit nimmt Winter besonders bei Frauen wahr, diese sollte auch durch das religiöse Ideal des „Leiden-auf-sich-Nehmens“ zusätzlich mental und spirituell gefördert werden. Auf den Punkt gebracht ging es darum, die Frauen an sich zugeordneten Attribute der Fürsorglichkeit, Selbstlosigkeit und Leidensfähigkeit im Religionsunterricht und in der Ausbildung zur Sozialhelferin oder Krankenschwester gezielt anzusprechen und weiter zu entwickeln. Auch die ehrenamtliche Tätigkeit von Frauen in diesen Bereichen sollte seiner Ansicht nach gefördert werden. Die Ähnlichkeiten zum ,josephinischen“ Gesundheitsund Sozialwesen könnten kaum deutlicher sein, nur handelt es sich hier nicht um den Pfarrer, sondern um die Gemeindehelferin.

Die Betonung der Bedeutung von Gérard van Swieten schließlich ist in den Publikationen von Erna Lesky eindeutig ein zentraler Punkt. Van Swieten war auch für ihre Vorgänger im Fach eine bedeutende Persönlichkeit, die Konstruktion des „Mythos van Swieten“ geht jedoch auf Lesky zurück und dürfte mit ihrer Rezeption von Eduard Winters Verständnis von „Josephinismus“ in Zusammenhang stehen. Die Ähnlichkeit ihrer Darstellung mit Winters Begriff „die Großen“ aus Wien ist nicht zu übersehen, etwa dann, wenn eine Kapitelüberschrift Der Große aus Wien lautet. ${ }^{85}$ Valjavec thematisiert wohl die „Kulturbringer" aus Wien, die ein Gesundheits- und Sozialwesen auch in entlegenen Gebieten aufgebaut hatten, erwähnt jedoch nicht die Rolle der Ärzte, die am Josephinum ausgebildet worden waren.

Hier stellt sich nun die Frage nach einer konkreten damnatio memoriae verschiedener Details und Personen sowie nach der gesellschaftlichen Bedeutung einer aus heutiger Sicht typischen Institution des „Josephinismus“, des Josephinums. Dieses Ausblenden ist, wie gezeigt wurde, an hochschulpolitische, aber auch weltanschauliche Verwerfungen im Vormärz rückzubinden. Zudem war, zumindest was die Medizin und die Naturwissenschaften betrifft, in der zweiten Hälfte des 19. und im beginnenden 20. Jahrhundert eine national ausgerichtete und durchaus auch antisemitische Tradition - unter anderem bei Anton von Rosas und Theodor Billroth - in hohem Ausmaß präsent, was entsprechende historiographische Zugänge und Themenfindungen ebenfalls beeinflusst haben kann.

Hinzu kommt, dass sozialgeschichtliche Perspektiven noch keinen Eingang in die Geschichtswissenschaft gefunden hatten. Dies betrifft das Gesundheits- und Sozialwesen, aber auch das Thema Bildung und soziale Mobilität, für die das Josephinum zumindest im 18. und frühen 19. Jahrhundert als beispielhafte Institution zu verstehen ist. Leskys einseitige Bewertung von Andreas Stifft ist hier aufschlussreich: Stifft kam "nur" aus bürgerlichen Verhältnissen und dass er auch medizinische Bücher verfasst hat, wird in einer Weise vermittelt, die ein solches Unterfangen geradezu als Anmaßung erscheinen lässt; zumal solch ein Mann auch noch sämtliche Ämter vertrat, die Gérard van Swieten einst innegehabt hatte.

85 LeSky, Gerhard van Swieten. Auftrag und Erfüllung, wie Anm. 15, 33. 


\section{EPILOG}

Assoziativ drängt sich eine Zusammenschau von Leskys Narrativ und den populären Geschichtskonstruktionen der frühen Zweiten Republik auf, wie sie in Ausstellungen, Themenschwerpunkten, kinematographischen Darstellungen und im Fernsehen greifbar wurden - etwa in der TV-Serie Der Kurier der Kaiserin. Maria Theresia wendet sich an ihren Sonderbeauftragten mit den Worten „Ich habe einen Auftrag für Sie“; dieser erfüllt den Auftrag in den folgenden 30 Minuten auf die ihm eigene draufgängerische Weise.

Diese Darstellung, die sich einem breiten Publikum eingeprägt hat, kann als eine Unterstützung des in den 1950er bis 1970er Jahren auch in publikumswirksamen Ausstellungen und über andere Informationsvektoren vermittelten Geschichtsbildes verstanden werden. ${ }^{86}$ Der von Lesky für ihren Aufsatz im von ihr mitherausgegeben Tagungsband Van Swieten und seine Zeit gewählte Titel - Auftrag und Erfüllung -, auf den sich auch der Titel dieses Beitrages bezieht, erschließt sich vor genau diesem Hintergrund als verklärende Geschichte eines „großen Mannes“ einer nur selektiv zu den Quellen vordringenden Medizinhistorikerin, inszeniert auf einer nicht weiter problematisierten ,josephinischen “ Bühne und gleichzeitig als Resultat einer im 19. Jahrhundert wurzelnden Anstrengung der Wiener Medizinhistoriographie, ihre josephinischen Wurzeln zu verbergen.

86 Thomas Wallnig / Johannes Frimmel / Werner Telesko (Hg.), 18th Century Studies in Austria, 1945-2010. Bochum 2011. 